\title{
Phospholipase C- $\gamma 2$ and Vav cooperate within signaling microclusters to propagate $B$ cell spreading in response to membrane-bound antigen
}

\author{
Michele Weber, ${ }^{1}$ Bebhinn Treanor, ${ }^{1}$ David Depoil, ${ }^{1}$ Hisaaki Shinohara, ${ }^{2}$ \\ Naomi E. Harwood, ${ }^{1}$ Masaki Hikida, ${ }^{2}$ Tomohiro Kurosaki, ${ }^{2}$ \\ and Facundo D. Batista ${ }^{1}$
}

'Lymphocyte Interaction Laboratory, London Research Institute, Cancer Research UK, London WC2A 3PX, England, UK
2Laboratory for Lymphocyte Differentiation, RIKEN Research Center for Allergy and Immunology, Tsurumi-ku, Yokohama,
Kanagawa 230-0045, Japan

$B$ cell receptor (BCR) recognition of membrane-bound antigen initiates a spreading and contraction response, the extent of which is controlled through the formation of signalingactive BCR-antigen microclusters and ultimately affects the outcome of B cell activation. We followed a genetic approach to define the molecular requirements of BCR-induced spreading and microcluster formation. We identify a key role for phospholipase $\mathrm{C}-\boldsymbol{\gamma} \mathbf{2}$ (PLC $\gamma_{2}$ ), Vav, B cell linker, and Bruton's tyrosine kinase in the formation of highly coordinated "microsignalosomes," the efficient assembly of which is absolutely dependent on Lyn and Syk. Using total internal reflection fluorescence microscopy, we examine at high resolution the recruitment of PLC $\gamma 2$ and Vav to microsignalosomes, establishing a novel synergistic relationship between the two. Thus, we demonstrate the importance of cooperation between components of the microsignalosome in the amplification of signaling and propagation of B cell spreading, which is critical for appropriate B cell activation.

CORRESPONDENCE

Facundo D. Batista:

facundo.batista@cancer.org.uk

Abbreviations used: BCAP, B cell adaptor for PI3K; Blnk, B cell linker; Btk, Bruton's tyrosine kinase; cSMAC, central supramolecular activation cluster; DIC, differential interference contrast; EGFP, enhanced GFP; GEF, guanine nucleotide exchange factor; HEL, hen egg lysozyme; IP, inositol 1,4,5triphosphate; IRM, interference reflection microscopy; IS, immunological synapse; PI3K, phosphoinositide 3-kinase; $\mathrm{PIP}_{2}$, phosphatidylinositol bisphosphate; $\mathrm{PKC} \beta$, protein kinase C- $\beta$; PLC $\gamma 2$, phospholipase C- $\gamma 2$; PLC $\gamma 2-L D$, PLC $\gamma 2$ with lipase-defective activity; SEM, scanning electron microscopy; SH2, Src homology 2; TIRFM, total internal reflection fluorescence microscopy; Vav1/2-KO, Vav1 and Vav2 knockout.
Naive B cells recognize antigen through their $\mathrm{BCR}$. The BCR is composed of membrane $\operatorname{IgM}$ and $\operatorname{IgD}$, responsible for the extracellular recognition of antigen, in complex with Ig $\alpha$ and $\operatorname{Ig} \beta$, which allow the transmission of intracellular signals through cytosolic immunoreceptor tyrosine-based activation motifs. Upon BCR engagement, tyrosine residues within these immunoreceptor tyrosine-based activation motifs are phosphorylated by Src family kinases, such as Lyn, which subsequently recruit and activate Syk (1-3). It has been demonstrated biochemically that activation of these kinases initiates the assembly of a multiprotein complex of adaptors and various signaling molecules at the plasma membrane, known as the signalosome $(4,5)$. These signaling platforms allow extensive coordination of and cross talk between intracellular signaling pathways involved in the regulation of critical cellular events such as the production of second messengers and changes in cytosolic calcium concentration. (1, 6-8). Ultimately,

$\overline{\text { The online version of this article contains supplemental material. }}$ these messengers determine the response that cells make to encountered antigen, such as cytoskeletal rearrangements or induction of gene expression (9). The particular signaling outcome appears to be dependent on the nature of the antigen (10) and on the stage of B cell development (11), which dictate the precise composition of the assembled signalosome $(1,12)$. However, the spatiotemporal dynamics of the signalosome have not been investigated.

$B$ cells encounter antigens in several different forms; however, it has been demonstrated that, in vivo, antigen is recognized predominantly in the form of immune complexes or directly on the surface of presenting cells such as follicular dendritic cells $(13,14)$, dendritic cells $(15,16)$, and macrophages (17-19). We have previously shown that the specific recognition of membrane-tethered antigen by BCR results in the dramatic rearrangement of molecules within the $\mathrm{B}$ cell membrane and the formation of an immunological synapse (IS) (20), similar to that observed in T cells $(21,22)$. The IS is composed of BCR-antigen accumulated into a 


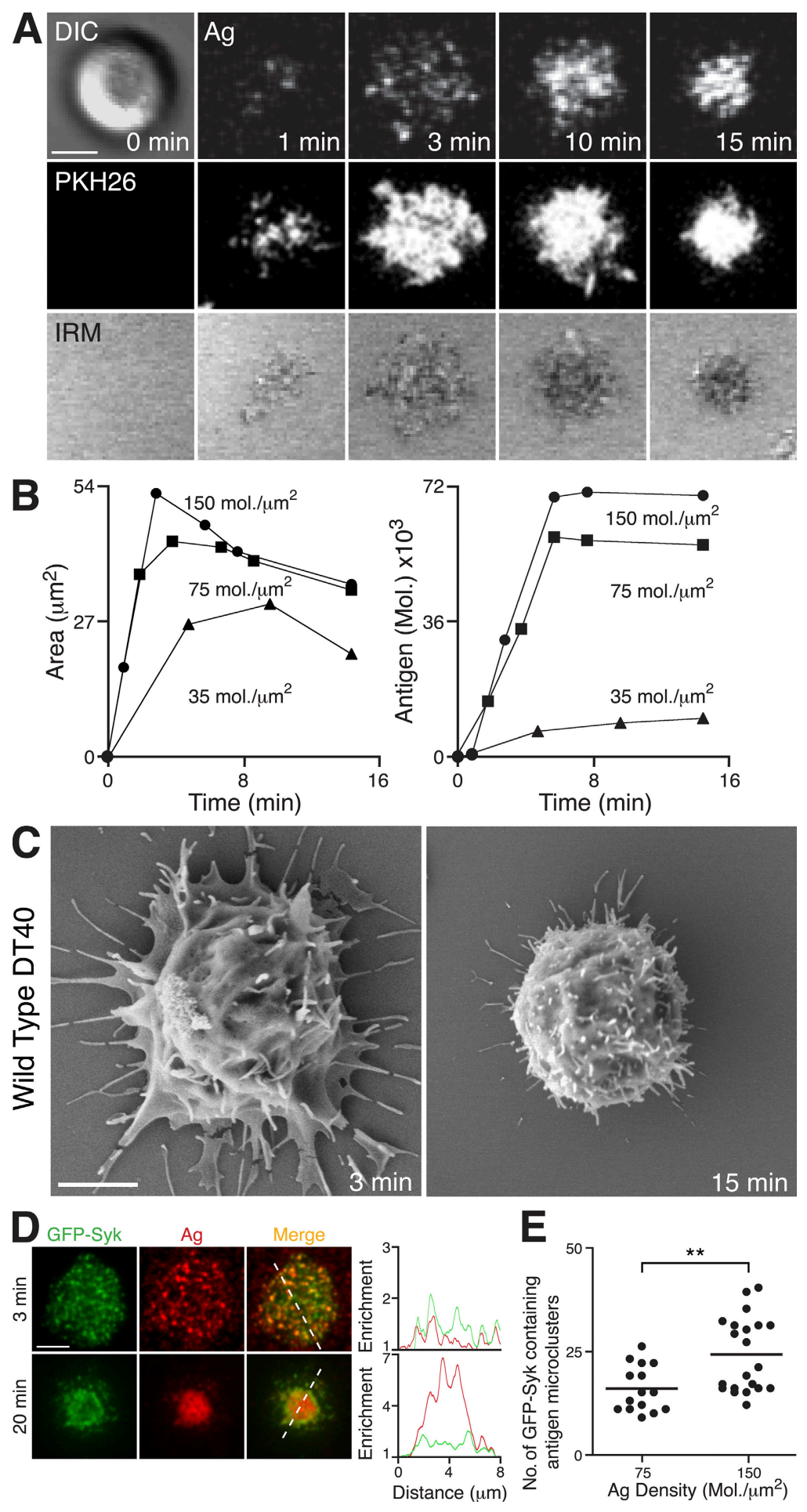

Figure 1. DT40 B cells as a model system to examine cellular responses to membrane-bound antigen. (A-C) DT40 B cells were settled onto bilayers containing anti-lgM as antigen $(\mathrm{Ag})$. ( $\mathrm{A}$, top) DIC (left) and confocal microscopy (right) visualizing antigen. ( $\mathrm{A}$, middle) Confocal microscopy visualizing B cell membrane (PKH26). (A, bottom) IRM visualizing contact area. Bar, $5 \mu \mathrm{m}$. (B) Bilayer antigen density was varied, as depicted, and (left) contact area by IRM and (right) antigen accumulation were quantified. (C) SEM images. Bar, $2 \mu \mathrm{m}$. (D and E) DT40 B cells expressing GFP-Syk (green) were settled on bilayers containing anti-lgM (red) as antigen (Ag). (D) TIRFM images. Relative fluorescence intensity plots of GFP-Syk and antigen are depicted by the diagonal dashed lines. Bar, $5 \mu \mathrm{m}$. (E) Quantification of GFP-Syk-containing antigen microclusters after $3 \mathrm{~min}$ at the indicated antigen densities. Data represent two independent experiments. The number of antigen microclusters containing GFP-Syk was counted in at least 15 cells of each cell type, representing a total of 779 microclusters. Mean numbers for high and low antigen density are $25 \pm 2$ and $17.4 \pm 1$, respectively. ${ }^{* *}, P<0.005$. Videos 1 and 2 are available at $h t t p: / / w w w . j e m . o r g / c g i / c o n t e n t / f u l l / j e m .20072619 / D C 1$. 
central supramolecular activation cluster (cSMAC) bordered by an outer ring of adhesion molecules in the peripheral SMAC. The IS provides a platform for B cell antigen accumulation, internalization, and subsequent presentation to $\mathrm{T}$ cells. The early events that occur upon B cell encounter of membrane-bound antigen, before formation of the mature IS, have been visualized by real-time fluorescence microscopy (23). B cells are induced to spread by extension of lamellipodia across an antigen-containing membrane surface and form BCR-antigen microclusters throughout the contact area, in parallel with an intracellular calcium response. The spreading response influences the outcome of B cell activation, as it functions to increase the number of antigen microclusters gathered and, consequently, the amount of antigen accumulated into the cSMAC during the contraction phase (23). This two-phase spreading and contraction response is dependent on the affinity and density of antigen, and requires both the initiation of intracellular signaling and reorganization of the cytoskeleton.

Classical biochemical techniques have been invaluable for studying the components of signaling pathways and provide an important foundation for studying interactions between isolated molecules and their assembly into functional signalosomes. Increasingly, however, single-cell fluorescence imaging techniques are being used to analyze the molecular dynamics of signaling cascades in their cellular context, offering new insights into the regulation and function of intracellular processes (24). Indeed, the discovery of the macromolecular organization of molecules at the IS was dependent on the visualization of proteins within the cellular context. The value of a fluorescence imaging approach is further illustrated by the description of signaling within receptor microclusters that temporally evolve to sustain signaling in the periphery of the IS (25-29). Such spatially resolved biochemistry is also important for assessing context-dependent functions of individual molecules, as demonstrated by the description of an essential but previously unidentified role for the co-stimulatory molecule CD19 in the recognition of membrane-bound antigen (29). Hence, it is of vital importance that the formation of a signalosome be investigated in terms of the spatiotemporal coordination of its component parts to extract biologically relevant and significant conclusions.

As the spreading and contraction response plays a key role in the determination of $\mathrm{B}$ cell fate, we have performed an extensive genetic dissection of this response in the DT40 $\mathrm{B}$ cell line to identify and characterize the spatiotemporal coordination of participating cellular effectors downstream of the BCR. In this study, we show that the tyrosine kinases Lyn and Syk act sequentially in the initiation of B cell spreading and demonstrate recruitment of Syk to antigen microclusters. A downstream intracellular signaling pathway involving Vav, phospholipase C- $\gamma 2$ (PLC $\gamma 2$ ), B cell linker (Blnk), and Bruton's tyrosine kinase (Btk) was found to be necessary for propagation of the spreading response, and we have verified the wider applicability of these findings in mouse splenic B cells.
In addition, we have used high resolution total internal reflection fluorescence microscopy (TIRFM) to visualize the dynamic recruitment of PLC $\gamma 2-$ GFP and Vav-GFP to numerous signaling antigen microclusters, and thus, redefine them as "microsignalosomes." From these studies, we suggest that PLC $\gamma 2$ and Vav act in concert to amplify the BCRantigen signal through the spreading response. This synergistic amplification ensures the gathering of antigen within the IS for extraction and presentation to $\mathrm{T}$ cells, ultimately influencing B cell activation.

\section{RESULTS \\ DT40 B cells as a tool for the genetic dissection of $B$ cell spreading}

We were interested in defining the dynamic assembly of the signalosome concerned with the initiation and propagation of B cell spreading. We sought to achieve this by using the chicken DT40 B cell line, as it has proven an exceptionally useful tool for large-scale assessment of the contribution of specific molecules to signaling pathways, including those that cannot be evaluated in knockout mice because of embryonic lethality (30). To validate the use of the DT40 B cell line as a model for examination of this cellular response, we used an adapted glass-supported planar lipid bilayer system containing biotinylated lipids, to which anti-chicken IgM as surrogate antigen was tethered via Alexa Fluor 633labeled streptavidin.

DT40 cells were found to spread and contract in response to membrane-bound antigen stimulation (Fig. $1 \mathrm{~A}$; and Video 1, available at http://www.jem.org/cgi/content/full/ jem.20072619/DC1), in a manner similar to that observed in mouse B cells (23). This was clearly seen by visualizing the distribution of antigen in the lipid bilayer, the fluorescent dye PKH26 within the cell membrane, and the contact area between cells and the bilayer using interference reflection microscopy (IRM; Fig. 1 A). The two-phase response exhibited the characteristic features observed in mouse B cells in terms of its dependence on antigen density and associated intracellular calcium flux (Fig. $1 \mathrm{~B}$ and Fig. S1). We have also used scanning electron microscopy (SEM) to visualize the extension of membrane processes of DT40 cells over the antigen-containing bilayer surface (Fig. $1 \mathrm{C}$ ). It has been recently established that antigen microclusters are the site of active signaling in primary mouse $\mathrm{B}$ cells, revealed by their rapid recruitment of GFP-Syk after BCR engagement (29). This recruitment was similarly observed in DT40 B cells (Fig. 1 D and Video 2) such that GFP-Syk was found in more than 70\% of antigen microclusters after contact with antigen-containing bilayers (not depicted). The number of GFP-Syk-positive antigen microclusters generated was dependent on the antigen density, consistent with the role of antigen microclusters as the basic units of signaling (Fig. 1 E). As observed previously (29), these GFP-Syk-containing microclusters were pushed out toward the periphery of the membrane extensions during the spreading phase before migrating toward the center of contact during contraction. 
Table I. B cell spreading and contraction response in DT40 B cell knockouts

\begin{tabular}{|c|c|c|c|}
\hline DT40 knockout & $\begin{array}{l}\text { Contact area } \\
\text { (\% of WT) }\end{array}$ & $\begin{array}{l}\text { Antigen aggregation } \\
\text { (\% of WT) }\end{array}$ & $\begin{array}{c}\text { No. of antigen microclusters } \\
\text { (\% of WT) }\end{array}$ \\
\hline Lyn-KO & $18 \pm 1.4^{* * *}$ & $40 \pm 2.1^{* * *}$ & $42 \pm 4^{* * *}$ \\
\hline Syk-KO & $18 \pm 1^{* * *}$ & $57 \pm 2.5^{* * *}$ & $36 \pm 4^{* * *}$ \\
\hline Lyn/Syk-KO & $13 \pm 0.9^{* * *}$ & ND & ND \\
\hline Blnk-KOa & $73 \pm 3^{* * *}$ & $98 \pm 3.3$ (ns) & $68 \pm 5^{* *}$ \\
\hline Blnk/Syk-KO & $16 \pm 1.5^{* * *}$ & ND & ND \\
\hline PLC $\gamma 2-K 0$ & $34 \pm 2.2^{* * *}$ & $45 \pm 2.4^{* * *}$ & $49 \pm 4^{* * *}$ \\
\hline Btk-KO & $52 \pm 3.2^{* * *}$ & $81 \pm 4.6^{*}$ & $69 \pm 3^{* *}$ \\
\hline Vav3-KO & $39 \pm 2.5^{* * *}$ & $63 \pm 7.7^{*}$ & $70 \pm 3^{* *}$ \\
\hline BCAP-KO & $76 \pm 5.6^{* *}$ & $129 \pm 8.8$ (ns) & ND \\
\hline PI3K-KO & $109 \pm 4.7(\mathrm{~ns})$ & $95 \pm 8.7$ (ns) & ND \\
\hline $\mathrm{IP}_{3} \mathrm{R}-\mathrm{KO}$ & $85 \pm 3.1^{* *}$ & $86 \pm 5.6^{*}$ & $71 \pm 6^{* *}$ \\
\hline PKC $\beta-K O$ & $88 \pm 3.8^{* *}$ & $82 \pm 6.8^{*}$ & $74 \pm 16^{* *}$ \\
\hline
\end{tabular}

B cells were settled on bilayers containing anti-IgM as antigen, and responses were visualized by confocal microscopy, IRM, or TIRFM. The responses of the various knockouts are classified by three parameters relative to WT: contact area $(3 \mathrm{~min})$, antigen aggregation (10 $\mathrm{min})$, and number of microclusters ( $3 \mathrm{~min})$. Data from at least two experiments are represented for each cell type, and each parameter was measured in $23-82$ cells. ${ }^{* * *}, P<0.0001$; ${ }^{* *}, P<0.005 ;{ }^{*}, P<0.05$. ND, not determined; ns, not significantly different. aVideo 8 (available at http://www.jem.org/cgi/content/full/jem.20072619/DC1) illustrates impaired spreading response in BInk-DT40 cells.

Collectively, these findings validate the use of DT40 $\mathrm{B}$ cells as an investigative tool in the genetic dissection of the signaling pathway and the dynamics of signalosome assembly required for the initiation and regulation of $\mathrm{B}$ cell spreading after stimulation with membrane-tethered antigen. Hence, we selected and analyzed a large panel of DT40 B cell knockouts for their ability to spread and generate signaling microclusters, as described in the previous paragraph for WT cells. The DT40 knockouts analyzed included those deficient in specific adaptors, kinases, and enzymes involved in BCR signaling, i.e., Lyn, Syk, Btk, Blnk, PLC $\gamma 2$, Vav3, phosphoinositide 3-kinase (PI3K), B cell adaptor for PI3K (BCAP), inositol 1,4,5-triphosphate receptors $\left(\mathrm{IP}_{3} \mathrm{Rs}\right)$, and protein kinase $\mathrm{C}-\beta$ (PKC $\beta$ ) (31). The results of the genetic screen are described in detail in the following Results sections and are summarized in Table I.

\section{Lyn and Syk initiate spreading and assembly of signaling microclusters}

To ascertain the role of Lyn and Syk, we used Lyn-KO and Syk-KO DT40 B cells to investigate the initiation of the spreading response after BCR engagement. DT40 B cells deficient either in Lyn or Syk were unable to initiate cell spreading upon settling on antigen-loaded bilayers (Fig. $2 \mathrm{~A}$ and Table I). The dark signal observed by IRM indicated that the Lyn-KO and Syk-KO DT40 B cells adhere to the bilayer as a result of engaging antigen, but their contact area remains constant at around $10 \mu \mathrm{m}^{2}$ (Fig. 2 B). As a consequence, these cells only accumulate antigen passively, resulting in a reduction in the total amount of antigen gathered (Fig. 2 B). Interestingly, both Lyn-KO and Syk-KO DT40 B cells displayed a more migratory phenotype than WT cells. These cells attempted movement across the bilayer while remaining attached through the BCR-antigen interaction (Fig. 2 C; and Video 3, available at http://www.jem.org/cgi/content/full/
jem.20072619/DC1), indicating that they are unable to initiate a "stop" signal after BCR engagement. Using SEM, we observed a dramatically different morphology of Lyn-KO and Syk-KO compared with WT DT40 B cells (compare Fig. 2 D with Fig. 1 C). Lyn-KO and Syk-KO DT40 B cells possess numerous elongated filopodia-like projections in place of the lamellipodial protrusions observed in WT cells, thus revealing underlying defects in the cytoskeleton and potentially offering an explanation for their impaired spreading.

Importantly, expression of GFP-Syk in Syk-KO DT40 B cells completely restored the spreading and contraction response seen in WT DT40 B cells, and allowed the generation of signaling microclusters (Fig. 2, E [left] and F). In contrast, expression of GFP-Syk in Lyn-KO DT40 B cells was not sufficient to restore spreading and did not allow the effective recruitment of GFP-Syk to antigen microclusters (Fig. 2, E [right] and F). Several antigen microclusters were able to form, even in the absence of Lyn or Syk (Fig. 2 E), indicating that antigen microcluster formation occurs before BCR signal transduction.

These results demonstrate the crucial role of the Src family kinase Lyn and Syk in initiating cell spreading after BCR engagement in DT40 B cells. The recruitment of Syk was dependent on the presence of Lyn, identifying the former kinase as acting downstream of the latter in the triggering of $\mathrm{B}$ cell spreading and the generation of signaling microclusters.

\section{PLC $\gamma 2$ recruitment to antigen microclusters is required for cell spreading}

The genetic screen of DT40 B cells identified a key role for PLC $\gamma 2$ in the propagation of the spreading response to membrane-bound antigen (Table I). Upon contact with the antigencontaining lipid bilayer, PLC $22-K O$ DT40 B cells formed a limited number of antigen microclusters (Fig. $3 \mathrm{~A}$; and Video 4, available at http://www.jem.org/cgi/content/full/ jem.20072619/DC1). IRM images indicate that PLC $\gamma 2-K O$ 
A
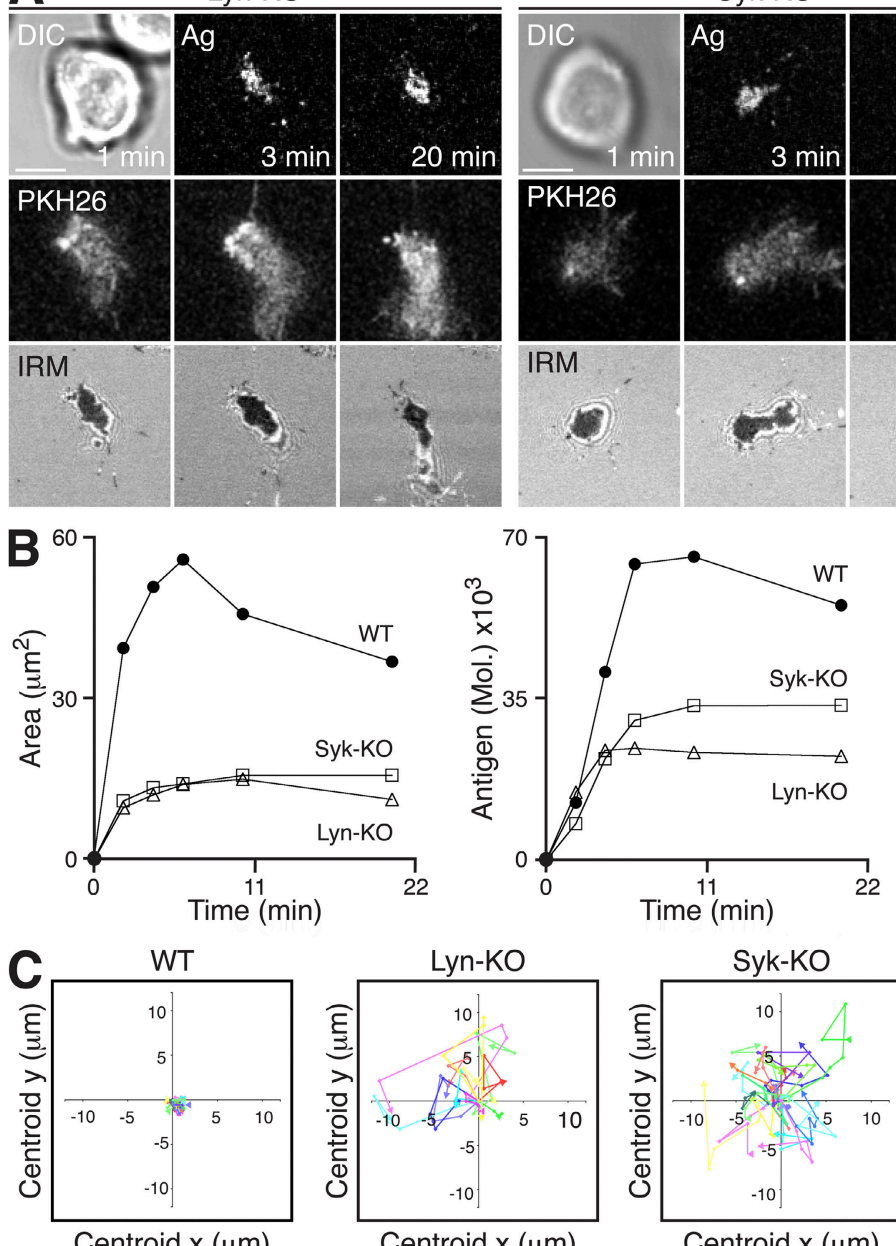

Centroid $\mathrm{x}(\mu \mathrm{m})$

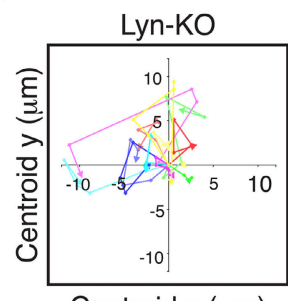

Centroid $\mathrm{x}(\mu \mathrm{m})$
Syk-KO

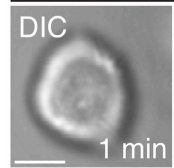

\section{PKH26}

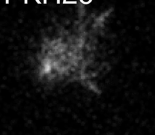

IRM
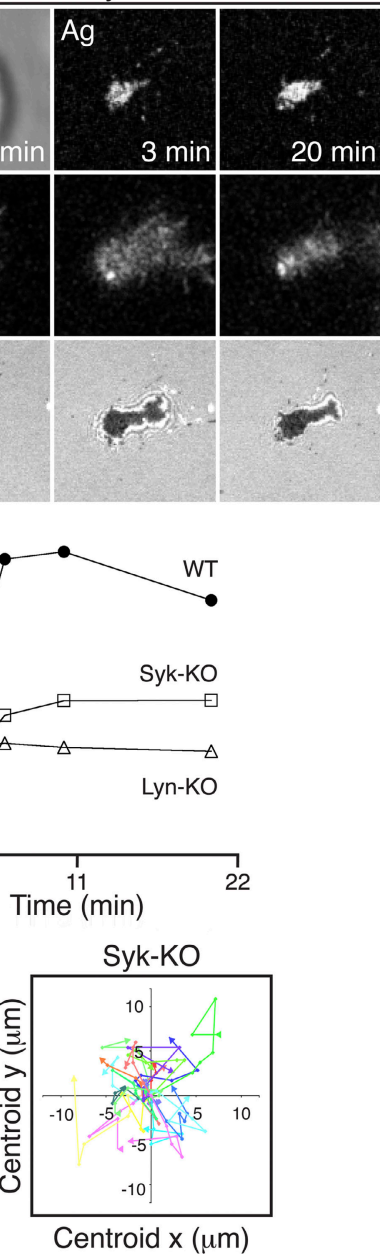

Centroid $\mathrm{x}(u \mathrm{~m})$

Lyn-KO
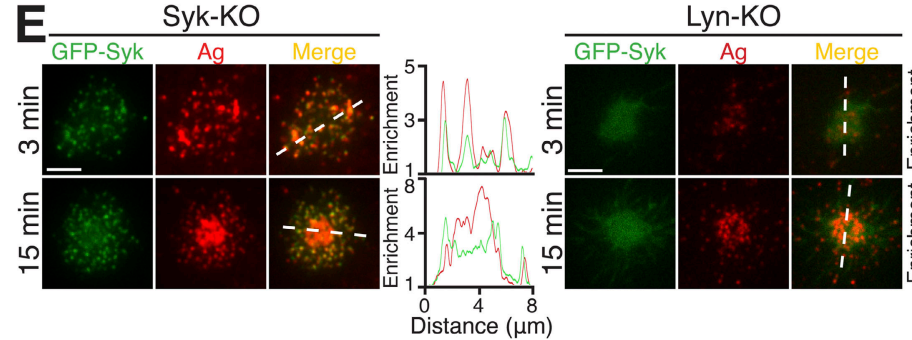

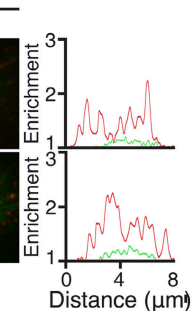

D
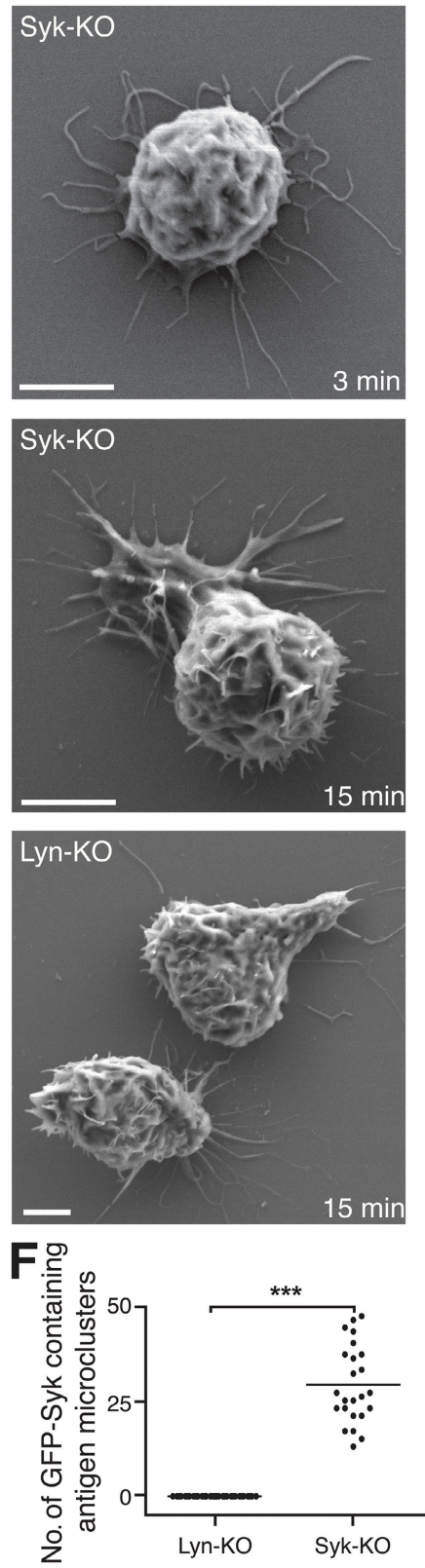

Figure 2. Lyn and Syk in the initiation of B cell spreading in response to membrane-bound antigen. (A-F) DT40 B cells were settled onto bilayers containing anti-lgM as antigen (Ag; red). (A) Lyn-KO and Syk-KO are shown. (top) DIC (left) and confocal microscopy (right) visualizing antigen. (middle) Confocal microscopy visualizing B cell membrane (PKH26). (bottom) IRM. Bars, $5 \mu \mathrm{m}$. (B) Quantification of (left) contact area by IRM and (right) antigen accumulation. (C) Two-dimensional tracking of individual WT, Lyn-KO, and Syk-KO cells. (D) SEM images. Bars, $2 \mu$ m. (E) TIRFM images of GFP-Syk (green) expressed in reconstituted Syk-KO and Lyn-KO. Relative fluorescence intensity plots to indicate the distribution of antigen and GFP-Syk are depicted by the diagonal dashed lines. Bars, $5 \mu \mathrm{m}$. (F) Quantification of GFP-Syk-containing antigen microclusters present after 3 min of interaction in reconstituted Syk-KO and Lyn-KO DT40 B cells. Data are from two experiments, and the number of antigen microclusters containing GFP-Syk was counted in 24-37 cells, representing a total of 698 microclusters. The mean numbers for reconstituted Syk-KO and Lyn-KO are $29 \pm 2$ and 0 , respectively. ${ }^{* * *}, P<$ 0.0001. Video 3 is available at http://www.jem.org/cgi/content/full/jem.20072619/DC1.

DT40 B cells are severely impaired in their ability to spread over time (Fig. 3, A and B). As a consequence, the total amount of antigen accumulated was reduced by up to $55 \%$ (Fig. $3 \mathrm{~B}$ and Table I). Interestingly, PLC $\gamma 2-\mathrm{KO}$ DT40 B cells also displayed an abnormal surface morphology (Fig. 3 C), similar to that seen in Lyn-KO and Syk-KO DT40 B cells (Fig. 2 E). Based on these observations, we assessed the role of PLC $\gamma 2$ in naive primary mouse $\mathrm{B}$ cells from a PLC $\gamma 2$ conditional 

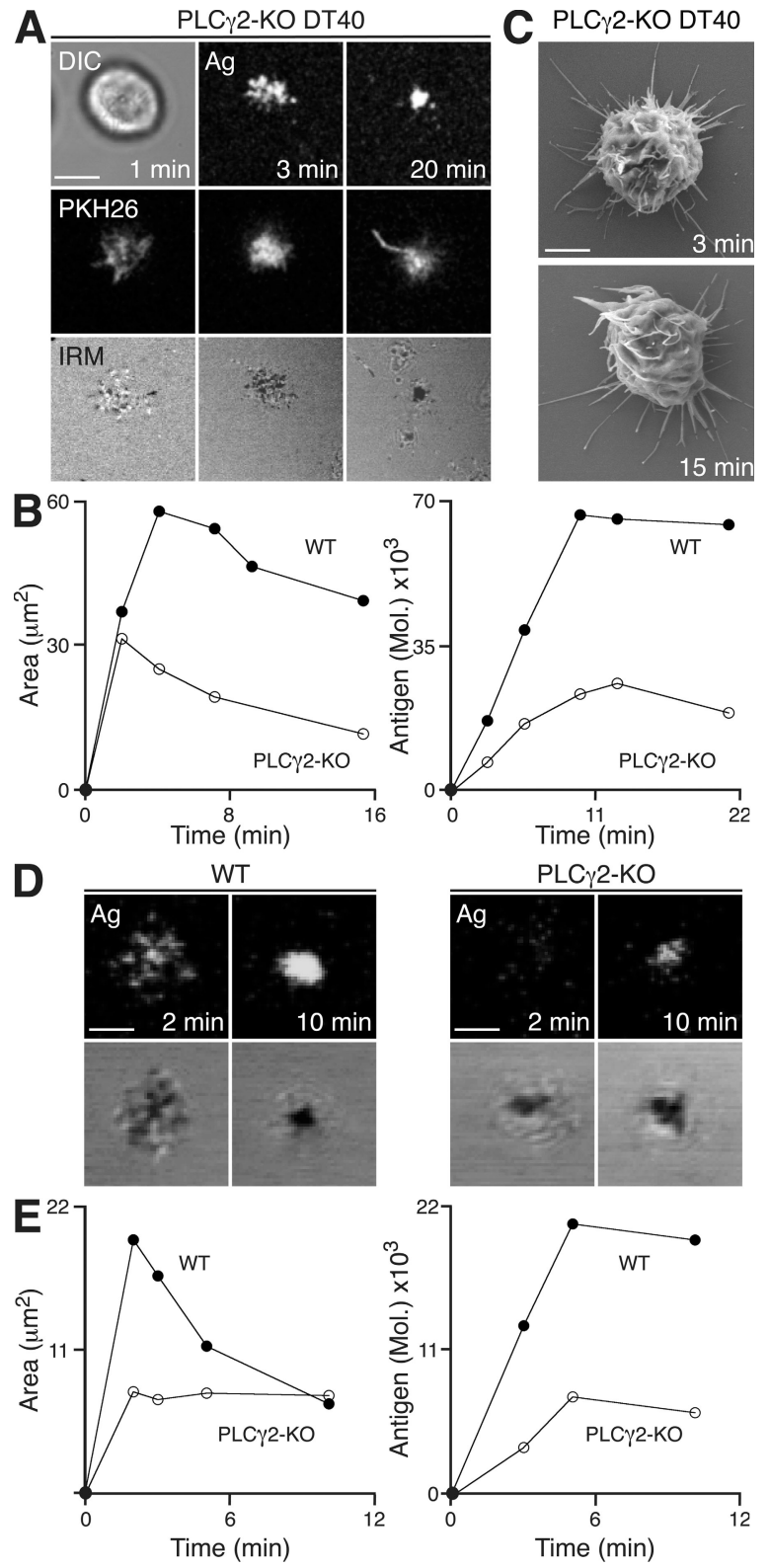

Figure 3. PLC $\gamma 2$ is critical for the propagation of cell spreading in response to membrane-bound antigen. (A-E) $B$ cells were settled onto bilayers containing anti-lgM $(\mathrm{A}-\mathrm{C})$ and anti- $\mathrm{\kappa}(\mathrm{D}$ and $\mathrm{E})$ as antigen $(\mathrm{Ag})$. (A-C) PLC 2 2-KO DT40 B cells. (A, top) DIC (left) and confocal microscopy (right) visualizing antigen. (middle) Confocal microscopy visualizing B cell

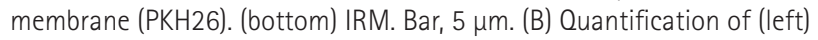
contact area by IRM and (right) antigen accumulation. (C) SEM images. Bar, $5 \mu \mathrm{m}$. (D and E) Naive WT (PLC $\gamma 2^{+/+} \mathrm{CD} 19 \mathrm{Cre}^{+/-}$) and PLC $2-\mathrm{KO}$ (PLC $\gamma 2^{\text {flox/flox } C D 19 C r e+/-}$ ) cells. (D, top) Confocal microscopy visualizing antigen distribution. (bottom) IRM. Bars, $2.5 \mu \mathrm{m}$. (E) Quantification of (left) contact area by IRM and (right) antigen accumulation. Video 4 is available at http://www.jem.org/cgi/content/full/jem.20072619/DC1.

knockout mouse using anti- $\mathrm{k}$ monoclonal antibody as a surrogate antigen. Primary naive mouse B cells were found to spread and contract after contact with lipid bilayer-containing antigen
(Fig. 3, D and E). However, PLC $\gamma 2$-deficient cells show a marked reduction in contact area and dramatic diminution in spreading, and as a result, a reduction in antigen accumulation (Fig. 3, D and E), thus demonstrating the wider applicability of our initial observations in the DT40 B cell line.

To investigate the localization of PLC $\gamma 2$ relative to antigen microclusters, we have generated a PLC $\gamma 2-$ GFP knock-in mouse on a PLC $\gamma 2-\mathrm{KO}$ background. This knock-in mouse allowed, for the first time, the dynamic visualization of the location of protein expressed at endogenous levels after BCR engagement. Splenic B cells from these knock-in mice were able to flux intracellular calcium upon BCR cross-linking, similar to WT B cells (Fig. S2, available at http://www.jem.org/ cgi/content/full/jem.20072619/DC1). Upon interaction with antigen-containing lipid bilayers, knock-in B cells formed antigen microclusters containing PLC $\gamma 2-G F P$ that multiplied throughout the contact area during spreading (Fig. 4 A; and Videos 5 and 6). PLC $\gamma 2-$ GFP remained associated with the microclusters as they fused and moved toward the center of cell contact during the contraction phase. A similar localization of PLC $\gamma 2-$ GFP to antigen microclusters was observed in PLC $\gamma 2-\mathrm{KO}$ DT40 B cells expressing PLCy2-GFP (Fig. 4 B and Video 7). These studies firmly establish the key role of PLC $\gamma 2$ in the propagation of B cell spreading through the dynamic recruitment of PLC $\gamma 2$ to antigen microclusters after BCR stimulation with membranebound antigen.

\section{Molecular requirements for PLC $\gamma 2$ function within microsignalosomes}

PLC $\gamma 2$ is a hydrolytic phosphodiesterase and contains two Src homology 2 (SH2) domains, which mediate binding to phosphotyrosine residues, as well as a lipase domain involved in catalyzing the generation of second messengers and, thus, propagating the initial signal $(32,33)$. Given the critical role of PLC $\gamma 2$ in B cell spreading, we used the genetic screen of DT40 B cells to identify the various signaling molecules involved in establishing the appropriate spatiotemporal coordination of PLC $\gamma 2$. Through this screen we have determined the molecular requirements for the correct localization and functioning of PLC $\gamma 2$ within signaling antigen microclusters, and as such redefine them as microsignalosomes.

The recruitment of PLC $\gamma 2$ to microsignalosomes is dependent on the initiation of signaling through Lyn and Syk, as would be expected given their critical role in the initiation of antigen microcluster assembly (Fig. 4, C and D). Subsequently, Blnk is thought to function as a key adaptor molecule in the recruitment of signaling molecules to the BCR signalosome (34-39). We observed that Blnk-KO DT40 B cells exhibited an impaired spreading response (Table I and Fig. S3, available at http://www.jem.org/cgi/content/full/ jem.20072619/DC1). However, the total amount of antigen accumulated in Blnk-KO DT40 was not significantly reduced compared with WT cells despite their impairment in effecting a regulated spreading response. We expect that the 

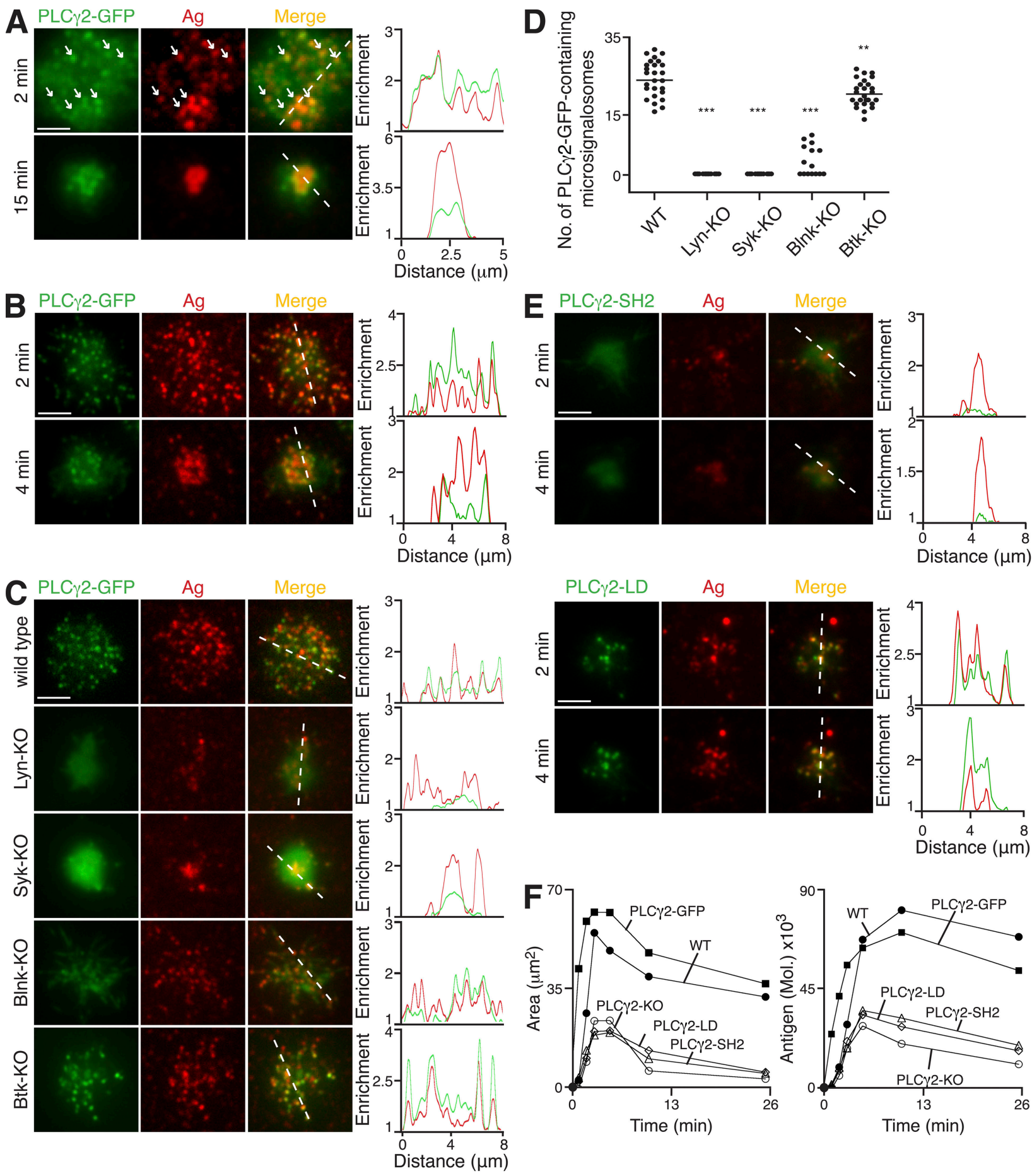

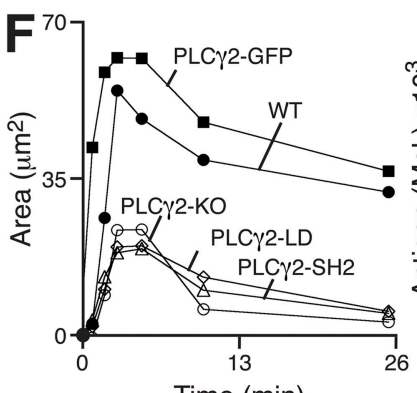

Time (min)

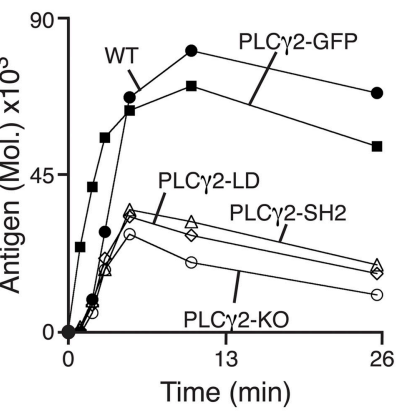

Figure 4. Molecular requirements for PLC $\gamma 2$ recruitment to antigen microclusters. (A-F) B cells were settled onto bilayers containing anti- $\kappa$ (A) and anti-IgM (B-F) as antigen ( $\mathrm{Ag}$; red). (A-C and E) TIRFM images. Relative fluorescence intensity plots to indicate the distribution of antigen and various PLC 2 2-GFP proteins (green) are depicted by the diagonal dashed lines. (A) Primary B cells from PLC 2 2-GFP knock-in mice. White arrows identify selected antigen microclusters containing PLC $\gamma 2-G F P$. (B) PLC $\gamma 2-K O$ DT40 B cells stably expressing PLC $\gamma 2-G F P$. (C) WT, Lyn-KO, Syk-KO, Blnk-KO, and Btk-KO DT40 $B$ cells expressing PLC $\gamma 2-G F P$ (green) after 3 min. (D) Quantification of PLC 2 2-GFP-containing microsignalosomes present in WT, Lyn-KO, Syk-KO, BInk-KO, and Btk-KO DT40 B cells after $3 \mathrm{~min}$. Data are representative of two experiments, and the number of antigen microclusters containing PLC $\gamma 2-\mathrm{GFP}$ was measured in 15-27 cells of each cell type, representing a total of 1,344 microclusters. Mean numbers are as follows: WT, $25 \pm 1$; Lyn-K0, 0; Syk-KO, 0; Blnk-KO, $3 \pm 1$; and Btk-KO, $21 \pm 1 .{ }^{* *}, \mathrm{P}<0.005 ;{ }^{* * *}, \mathrm{P}<0.0001$. (E) PLC $\gamma 2-\mathrm{KO}$ DT40 B cells stably expressing (top) PLC $\gamma 2-\mathrm{GFP}$ with mutated SH2 domains (PLC $2-S H 2)$ or (bottom) PLC 2 2-LD-GFP (PLC 2 2-LD). (F) Quantification of (left) contact area by confocal microscopy and (right) antigen accumulation. Bars: (A) $2.5 \mu \mathrm{m} ;(\mathrm{B}, \mathrm{C}$, and E) $5 \mu \mathrm{m}$. Videos 5-7 are available at http://www.jem.org/cgi/content/full/jem.20072619/DC1. 
A

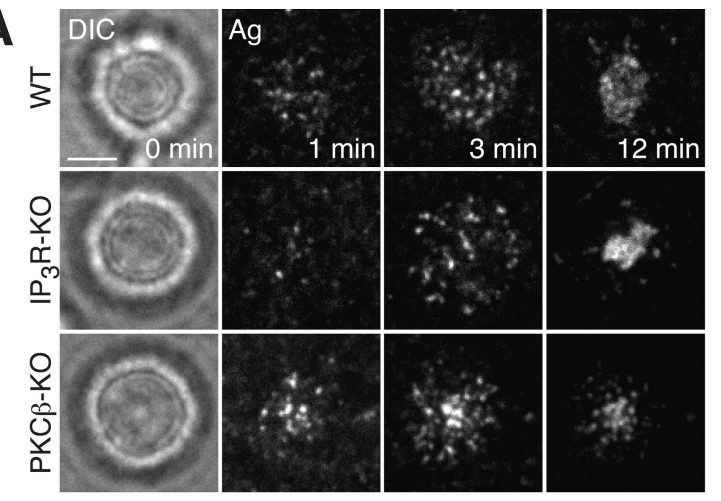

B
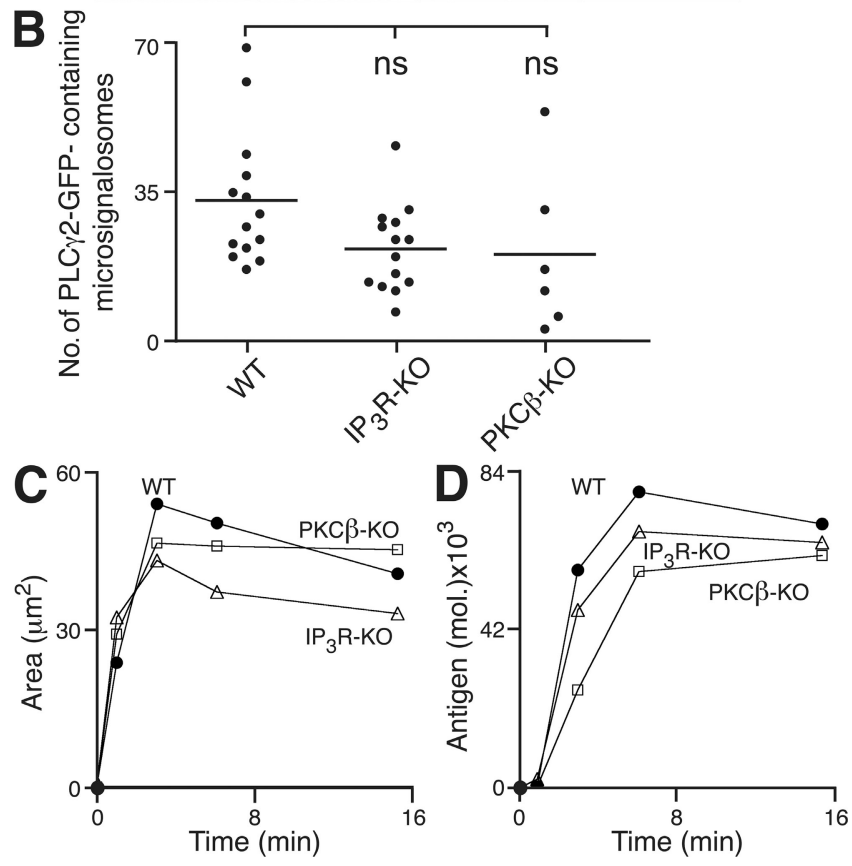

Figure 5. PLC $\gamma 2-$ mediated $B$ cell spreading and antigen aggregation in the absence of $I P_{3} R$ or PKC $\beta$. (A-C) WT, IP $R-K O$, or PKC $\beta-K O$ DT4O $B$ cells were settled onto bilayers containing anti-IgM as antigen ( $\mathrm{Ag})$. (A) Brightfield (left) and TIRFM (right) visualizing antigen. Bar, $5 \mu \mathrm{m}$. (B) Quantification of PLC $\gamma 2-G F P$-containing microsignalosomes present after $3 \mathrm{~min}$. Data are representative of two experiments, and the number of microsignalosomes was measured in 6-14 cells, representing a total of 892 microsignalosomes. Mean numbers are as follows: WT, $33 \pm 4 ; \mid P_{3} R-K O, 22 \pm 3$; and PKC $\beta-K O, 21 \pm 8$. ns, not significantly different. (C) Quantification of (left) contact area by confocal microscopy and (right) antigen accumulation.

antigen accumulation observed in these cells occurred as a result of engagement and transport of antigen through the numerous elongated filopodia that they display (Video 8). Blnk-KO DT40 B cells expressing PLC $\gamma 2-G F P$ rapidly formed antigen microclusters after their settling onto lipid bilayers containing antigen; however, very few of these microclusters were enriched in PLC $\gamma 2-$ GFP (Fig. 4, C and D). This suggests that in the absence of Blnk there is only a very transient association of PLC $\gamma 2$ within microsignalosomes, and as a consequence, the efficiency of these few signalosomes to propagate spreading is markedly reduced. In addi- tion, through the expression of PLC 22 with mutated SH2 domains in PLC $\gamma 2-K O$ DT40 B cells, we have established that PLCy2 recruitment is dependent on its SH2 domains (Fig. 4, E and F). These observations emphasize the importance of Lyn and Syk in the initiation of the signaling assembly, as well as the requirement of Blnk- and SH2-mediated recruitment of PLC 22 in the formation of effective microsignalosomes able to propagate $\mathrm{B}$ cell spreading.

Btk has been shown previously to be involved in the regulation of PLC $\gamma 2$ lipase activity (40-42), and we observed that Btk-KO DT40 B cells exhibited an impaired spreading response (Table I and Fig. S3). The recruitment of PLC $\gamma 2-$ GFP to microsignalosomes was observed in Btk-KO DT40 $\mathrm{B}$ cells at levels similar to the WT (Fig. 4, C and D), indicating that Btk is not essential for their assembly. Given the role of Btk in PLC $\gamma 2$ activation, we have examined the requirement for the enzymatic activity of PLC $\gamma 2$ within microsignalosomes. To do this, we have expressed PLC $\gamma 2-G F P$ with lipase-defective activity (PLC $\gamma 2-\mathrm{LD}-\mathrm{GFP}$ ) in PLC $\gamma 2-\mathrm{KO}$ DT40 B cells. PLC $\gamma 2-L D-G F P$ was found to be recruited in the same manner as observed for PLCy2-GFP; however, the recruitment alone, as demonstrated in the Btk-KO cells, was not sufficient to restore spreading (Fig. 4, E and F). Collectively, these data demonstrate that the assembly of the microsignalosome perse is not sufficient to propagate B cell spreading, as this response requires the correct functioning of all component parts.

The lipase activity of PLC $\gamma 2$ generates $\mathrm{IP}_{3}$ and diacylglycerol, and thus through calcium- and PKC $\beta$-mediated pathways, regulates a multitude of cellular functions (33). As B cell spreading depends on the lipase activity of PLC $\gamma 2$, we examined potential effectors for PLC $\gamma 2$. Interestingly, we found that DT40 B cells lacking all three types of $\mathrm{IP}_{3}$ receptors or PKC $\beta$ were able to spread and generate microsignalosomes with a small but reproducible reduction compared with WT cells (Fig. 5 and Table I). However, such a reduction is much less prominent than that observed in PLC $\gamma 2-$ KO DT40 B cells. As DT40 B cells lacking all three types of $\mathrm{IP}_{3}$ receptors are unable to release calcium from intracellular stores (43), this suggests that propagation of intracellular signaling through PLC $\gamma 2$ to stimulate spreading does not exclusively rely on $\mathrm{IP}_{3}$-mediated changes in intracellular calcium. However, as there appeared to be a short delay in the onset of antigen accumulation in the cSMAC in these cells, it may be that $\mathrm{IP}_{3}$ receptors and $\mathrm{PKC} \beta$ are involved in the regulation of the contraction phase of the cellular response.

Thus, we have demonstrated that the active recruitment to and functioning of PLC $\gamma 2$ within microsignalosomes is dependent both on the $\mathrm{SH} 2$ domains and lipase activity of PLC $\gamma 2$, and on Blnk and Btk. Hence, the correct formation of and communication within microsignalosomes is critical for the coordination of BCR-induced spreading. Intriguingly, we observe that propagation of B cell spreading through PLC 2 appears to operate through a mechanism that is not solely dependent on either $\mathrm{IP}_{3}$-mediated calcium release or $\mathrm{PKC} \beta$. 


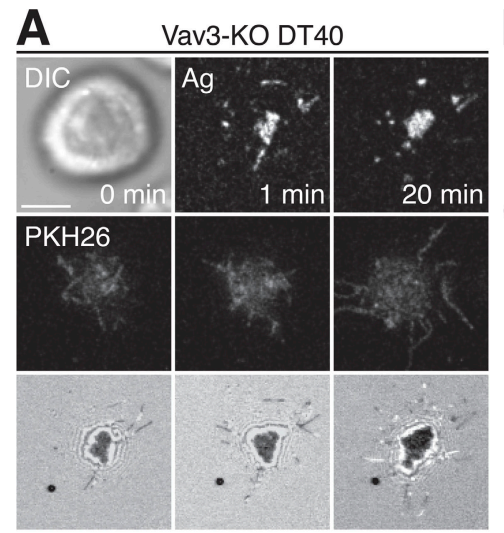

B
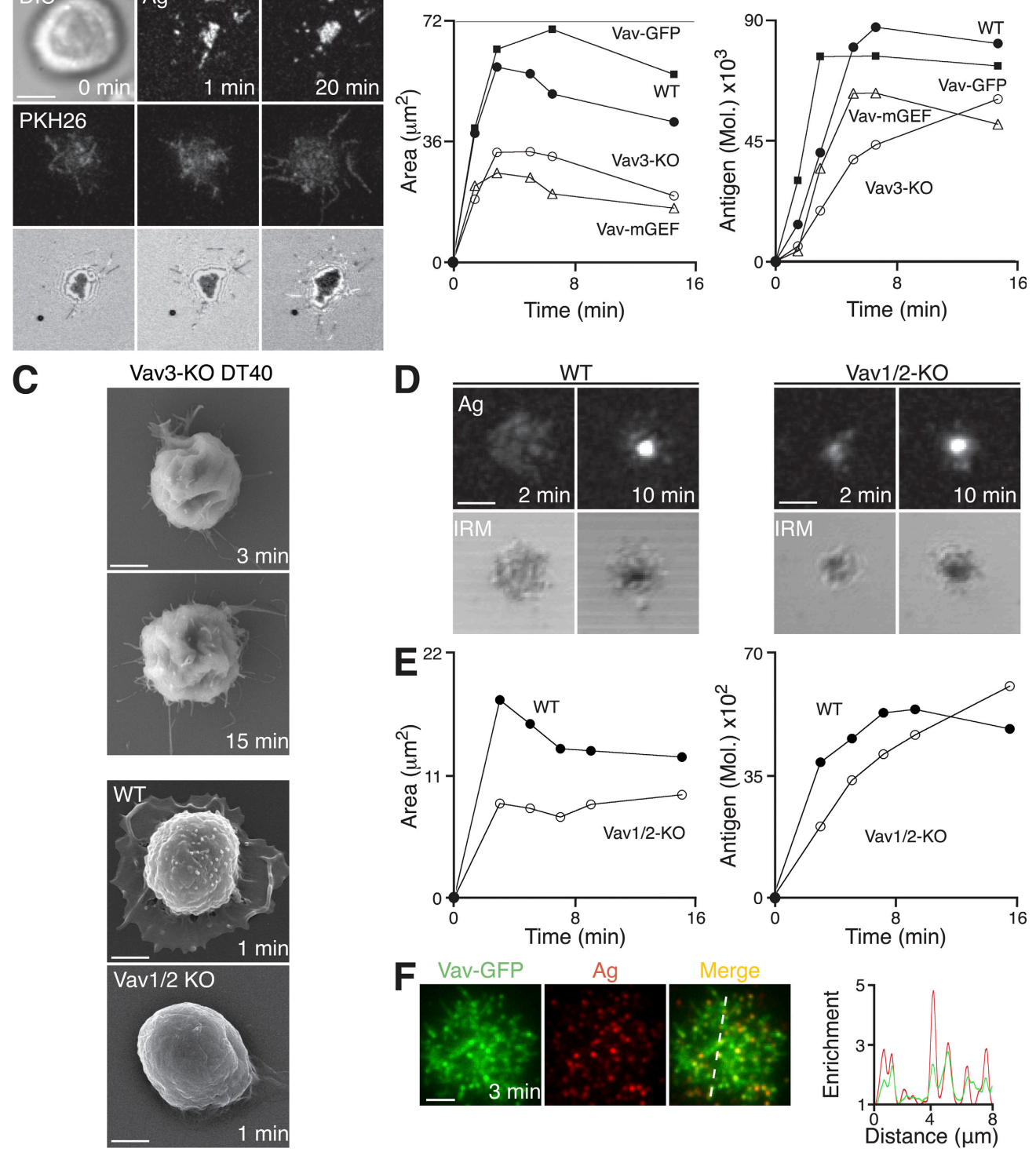

Figure 6. Vav is required for B cell spreading. (A-F) B cells were settled onto bilayers containing anti-lgM and HEL as antigen (Ag) for DT40 and primary B cells, respectively. (A) Vav3-KO DT40 B cells. (top) DIC (left) and confocal microscopy (right) visualizing antigen. (middle) Confocal microscopy visualizing B cell membrane (PKH26). (bottom) IRM. Bar, 5 mm. (B) WT, Vav3-KO, Vav3-KO expressing Vav-GFP, and Vav3-KO expressing Vav with mutated GEF activity DT40 B cells. Quantification of (left) contact area by IRM and (right) antigen accumulation. (C) SEM images. Bars, $2 \mu m$. (D and E) Primary MD4-HEL-Tg (WT) and Vav1/2-KO HEL-Tg (Vav1/2-KO) splenic B cells. (D, top) Confocal microscopy visualizing antigen. (bottom) IRM. Bars, $2.5 \mu \mathrm{m}$. (E) Quantification of (left) contact area by IRM and (right) antigen accumulation. (F) TIRFM images of DT40 B cells expressing Vav-GFP (green) after 3 min. Bar, $5 \mu \mathrm{m}$. A relative fluorescence intensity plot to indicate the distribution of Vav-GFP (green) and antigen (red) is depicted by the diagonal dashed line. Video 9 is available at http://www.jem.org/cgi/content/full/jem.20072619/DC1.

\section{Vav recruitment to microsignalosomes is required for spreading}

As we have observed that PLC $\gamma 2$ is critical for the propagation of $\mathrm{B}$ cell spreading through a mechanism not solely dependent on intracellular calcium signaling, we used our genetic screen of DT40 B cell knockouts to identify molecules that may influence PLC $\gamma 2$ recruitment to or activity within microsignalosomes. We found that PI3K and the as- sociated adaptor BCAP were largely dispensable for spreading in DT40 cells, as PI3K-KO and BCAP-KO B cells exhibited behavior similar to that seen in WT cells (Table I and Fig. S3). However, the genetic screen of DT40 B cells identified Vav3 as playing a critical role in the B cell spreading response. In DT40 B cells, Vav3 is the predominant member of the Vav family expressed, as demonstrated previously, by the significant reduction in the ability of Vav3-KO 
DT40 B cells to flux calcium (44). Vav3-KO DT40 B cells formed a limited number of antigen microclusters on contact with antigen-containing lipid bilayers but were severely impaired in their ability to spread over time and exhibited a reduction in antigen accumulation (Fig. 6, A and B; and Video 9). Using SEM, we have also observed an unusual morphology of Vav3-KO cells compared with WT B cells (Fig. 6 C). These cells seem to lack the capacity to form and extend membrane processes, indicating severe cytoskeletal abnormalities. Thus, we have investigated the role of Vav in naive primary mouse B cells using mice deficient in Vav1 and Vav2 (Vav1/2-KO) that were crossed with MD4 transgenic mice, such that they express BCR specific for hen egg lysozyme (HEL) (45). In contrast to DT40 B cells, it has been shown previously that both Vav1 and Vav2 are required to mediate Vav family protein function in mouse B cells (46).

Vav1/2-KO B cells show a marked reduction in B cell spreading when settled on lipid bilayers containing HEL as antigen (Fig. 6, C-E), and the accumulation of antigen in these cells was impaired during the initial stages of the cellular response (Fig. 6 E). However, at later time points, we observed that Vav1/2-KO B cells appear to accumulate more antigen than WT B cells. As Vav proteins are known to play a key role in the regulation of cytoskeleton rearrangements, we expect that the deletion of Vav in these cells results in major cytoskeletal irregularities, potentially influencing cell contraction and also antigen extraction, such that cells appear to continue to aggregate antigen for longer than WT cells. Hence, it is clear that Vav proteins play a key role in the regulation of spreading and antigen gathering in mouse $\mathrm{B}$ cells.

We have used high resolution TIRFM to investigate the localization of Vav-GFP in DT40 B cells in response to stimulation with membrane-bound antigen. Expression of VavGFP was sufficient to restore the initiation of the spreading response of Vav3-KO B cells in response to BCR stimulation (Fig. 6 B). It is known that Vav family proteins act as guanine nucleotide exchange factors (GEFs) for RhoGTPases in the remodelling of the actin cytoskeleton (47). Because expression of Vav with mutated GEF activity in Vav3-KO B cells was not sufficient for the restoration of spreading (Fig. 6 B), it is clear that this role of Vav depends on its GEF activity for subsequent activation of effectors. Overexpression of VavGFP in Vav3-KO B cells led to the maintenance of the contact area after maximal spread of these cells. This observation indicates that in addition to its role in the initiation of the cell-spreading response, the regulation of Vav activity may be important in the subsequent onset of contraction. As expected from its critical role in the BCR-proximal events that regulate spreading and contraction, Vav-GFP was indeed recruited to microsignalosomes (Fig. 6 F).

Thus, we have identified a critical role for Vav in the initiation of the spreading response both in DT40 and mouse $\mathrm{B}$ cells after stimulation with membrane-bound ligands. The role of Vav in this response involves its recruitment to microsignalosomes and is dependent on its activity as a GEF for intracellular effectors.

\section{Concerted action of PLC $\gamma 2$ and Vav in B cell spreading}

As PLC $\gamma 2$ and Vav are both recruited to the microsignalosome on BCR stimulation and function to propagate B cell spreading in response to membrane-bound antigen stimulation, we were keen to further characterize the role of and identify any relationship between these two important regulators. To do this, we expressed GFP-labeled Vav and PLC $\gamma 2$ in DT40 B cells deficient in PLC $\gamma 2$ and Vav3, respectively.

Expression of Vav-GFP in PLC $\gamma 2-K O$ DT40 B cells was not sufficient for restoration of the contact area or accumulation of antigen seen in WT B cells. However, the recruitment of Vav-GFP to microsignalosomes was observed (Fig. 7, A and B), demonstrating that the formation of microsignalosomes of altered composition, lacking PLC $\gamma 2$, is not sufficient to enable the spreading response. Similarly, the converse combination of expression of PLC 2 -GFP in Vav3-KO DT40 B cells was not sufficient for the restoration of the spreading, though PLC $\gamma 2$ was found to be recruited to microsignalosomes (Fig. 7, C and D). It is clear that although PLC $\gamma 2-G F P$ or Vav-GFP is recruited to microsignalosomes in the absence of the other, the total number of microsignalosomes formed is greatly reduced compared with WT DT40 B cells (Fig. 7, B and D). Interestingly, the relative frequencies of microsignalosomes containing PLC $\gamma 2-G F P$ or Vav-GFP in the Vav3-KO or PLC $\gamma 2-\mathrm{KO}$ cells, respectively, was also found to be reduced compared with WT DT40 B cells (Fig. 7 E). This observation indicates that PLC $\gamma 2$ and Vav influence the recruitment and retention of each other, suggesting cooperation between these two critical components of the microsignalosome. Thus, in the absence of either PLC $\gamma 2$ or Vav3, there is greater heterogeneity in terms of the composition and signaling competence of the microsignalosome population. We suggest that PLC $\gamma 2$ and Vav play distinct roles, proximal to signaling through the BCR, both of which are crucial for initiation of $\mathrm{B}$ cell spreading.

Collectively, these results demonstrate that PLC $\gamma 2$ and Vav cooperate to shape B cell responses to membrane-bound antigen. Both PLC $\gamma 2$ and Vav are absolutely required, in concert, for the development of the characteristic spreading response, allowing propagation of BCR signaling through the generation of sufficient numbers of microsignalosomes.

\section{DISCUSSION}

The spreading and contraction of $\mathrm{B}$ cells in response to membrane-bound antigen has been shown to determine the amount of antigen accumulated in the CSMAC and thereby influence the outcome of B cell activation. Through a detailed genetic dissection of the intracellular pathway activated after BCR engagement, we have demonstrated the requirement for the initiation of this response on the Src kinase Lyn and the subsequent recruitment of Syk to antigen microclusters. We have also identified a key role for PLC 2 2, Vav, Btk, and Blnk in the propagation of intracellular signaling and effecting the spreading response. Surprisingly the pathway identified in this study operates via a mechanism that does not 

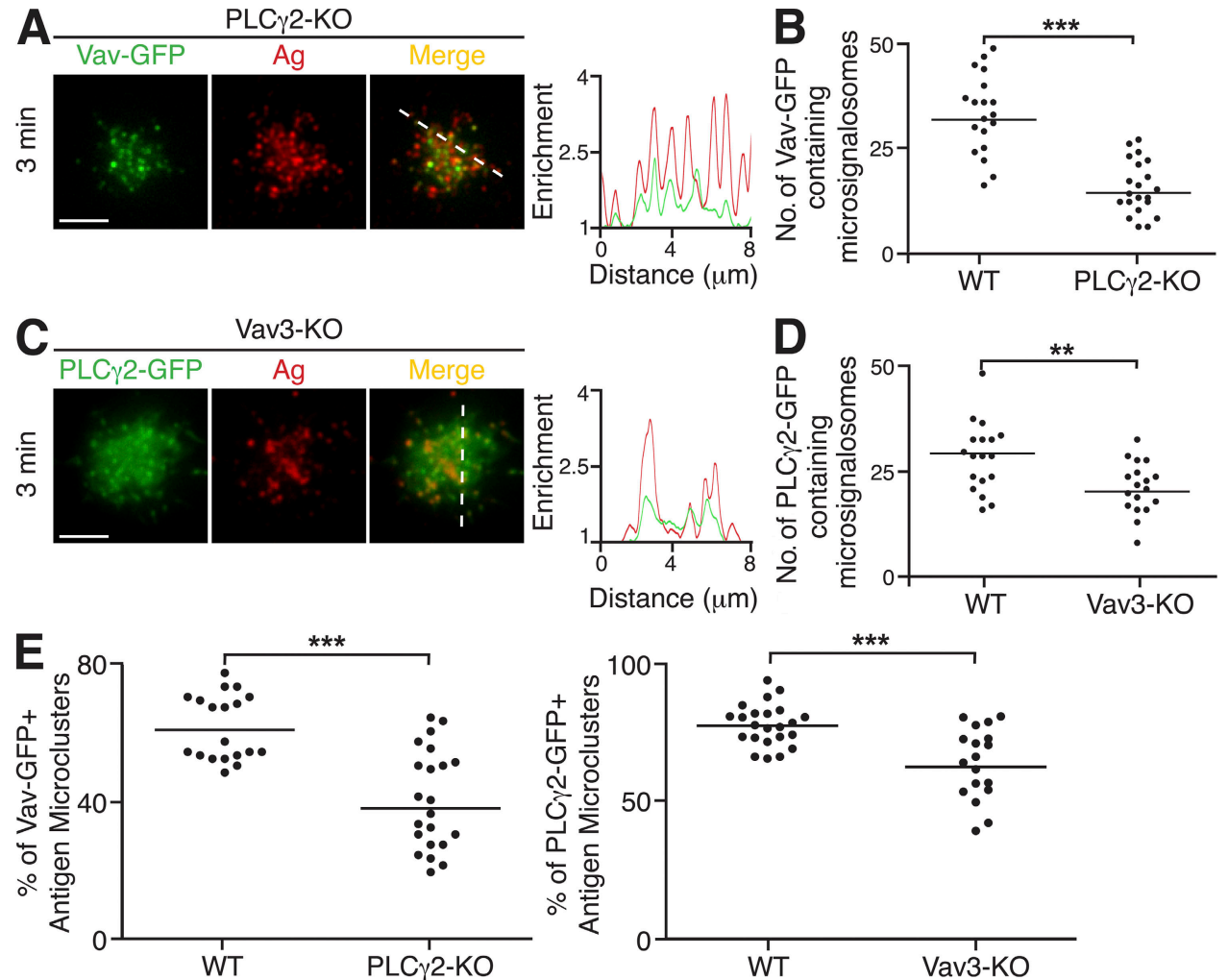

Figure 7. PLC $\gamma 2$ and Vav act in concert in the coordination of $B$ cell spreading in response to membrane-bound antigen. (A-E) $B$ cells were settled onto bilayers containing anti-IgM as antigen (Ag; red) and were analyzed after 3 min. (A and B) PLC 2 2-KO DT40 B cells expressing Vav-GFP (green). (A) TIRFM images. A relative fluorescence intensity plot to indicate the distribution of Vav-GFP and antigen is depicted by the diagonal dashed line. Bar, 5 um. (B) Quantification of Vav-GFP-containing microsignalosomes. Data are representative of two experiments, and the number of antigen microclusters containing Vav-GFP was measured in 15-20 cells of each type, representing a total of 934 microsignalosomes. Mean numbers in WT and PLC $\gamma 2-K 0$ are $36 \pm 3$ and $18 \pm 2$, respectively. ${ }^{* *}, P<0.001$. (C and D) Vav3-KO DT40 B cells expressing PLC $\gamma 2-G F P$ (green). (C) TIRFM images. A relative fluorescence intensity plot to indicate the distribution of PLC $\gamma 2-G F P$ and antigen is depicted by the diagonal dashed line. Bar, $5 \mu \mathrm{m}$. (D) Quantification of PLC 2 2-GFP-containing microsignalosomes. Data are representative of two experiments, and the number of microsignalosomes containing PLC $\gamma 2-G F P$ was measured in 18-23 cells of each cell type, representing a total of 1,061 microsignalosomes. Mean numbers in WT and Vav3-KO are $32 \pm 3$ and $22 \pm 6$, respectively. ${ }^{*}, \mathrm{P}<0.005$. (E) Quantification of (left) Vav-GFP-containing antigen microclusters in WT and PLC $\gamma 2-K 0$ DT40 B cells and (right) PLC $\gamma 2-$ GFP-containing antigen microclusters in WT and Vav3-KO DT40 B cells, expressed as a percentage of total antigen microclusters present. Data are representative of two experiments, and the percentage of antigen microclusters was measured in 15-23 cells of each cell type, representing a total of 1,476-1,811 antigen microclusters. (left) Mean percentages in WT and PLC $\gamma 2-K O$ are $62 \pm 3$ and $43 \pm 2$, respectively. ${ }^{* *}$, $P<0.001$. (right) Mean percentages in WT and Vav3-KO are $78 \pm 2$ and $64 \pm 3$, respectively. ${ }^{* *}, P=0.0001$.

exclusively rely on $\mathrm{IP}_{3}$ receptors or $\mathrm{PKC} \beta$ and is independent of PI3K and BCAP in DT40 cells. Using high-resolution single-cell imaging, we have been able to move away from the classical static view of the signalosome described previously $(4,5)$ toward a description of the spatiotemporal coordination of microsignalosomes in real time. As such, we have observed the dynamic localization of PLC $\gamma 2$ and Vav to antigen microclusters to redefine them as microsignalosomes competent for the propagation of intracellular signaling. The recruitment of PLC $\gamma 2$ is absolutely dependent on its $\mathrm{SH} 2$ domains, whereas efficient microsignalosome assembly and propagation also require the functioning of its constituents to mediate $\mathrm{B}$ cell spreading. In addition, we have demonstrated the importance of the synergistic action of PLC $\gamma 2$ and Vav, within microsignalosomes, in the coordination of the spreading response triggered by membrane-tethered antigen stimulation.
This study provides genetic evidence for the role of the Src family kinases in the initiation of the cellular response and the formation of microsignalosomes in B cells. As Lyn is the predominant Src family kinase expressed in DT40 B cells (48), we were able to identify and isolate a role for this particular kinase. However, as mouse B cells express several additional Src family kinases (49), it is possible that a degree of redundancy exists in the initiation of BCR signaling in mouse cells. The complete abrogation of spreading in Lyn-deficient DT40 B cells is consistent with the inhibition of BCR-induced spreading after treatment of mouse B cells with PP2, a Src kinase inhibitor (23). The presence of Lyn is absolutely required for the recruitment of Syk in DT40 B cells, in agreement with our previous observations in a mouse cell line expressing a chimeric signaling-deficient BCR (29) and with the lack of ZAP-70 recruitment in PP2-treated T cells $(25,27)$. 
Although Lyn and Syk are necessary for the propagation of BCR-induced spreading, antigen microclusters are able to form in their absence. This provides further evidence for the existence of antigen microclusters in the absence of signaling, and thus, it seems likely that they are formed as a result of diffusion trapping $(26,29)$.

Following membrane proximal signaling events initiated upon BCR engagement, we have found that B cells use a limited number of key proteins as molecular conduits to rapidly translate receptor signals into a cytoskeletal response. We have identified PLC $\gamma 2$ and Vav as the major coordinators of the $\mathrm{B}$ cell spreading response and have demonstrated their localization in microsignalosomes. These assemblies require the presence of adaptor molecules, which contain distinct and specific phosphorylation sites that form docking sites for several effector molecules and function to support interactions in a multimolecular complex (35). We demonstrated in this study that Blnk functions in the organization of microsignalosomes through the recruitment of Vav and PLC $\gamma 2$. Vav is the only GEF with SH2 domains identified at present and may be regulated through interaction with phosphorylated residues in Blnk (35) or the coreceptor CD19 (50). Despite this important role for Blnk in microsignalosome assembly, we observed a limited degree of PLC $\gamma 2$ recruitment in Blnk-KO DT40 B cells, suggesting that there may be secondary mechanisms for PLC $\gamma 2$ recruitment to the membrane. Such an observation is in agreement with the identification of 3BP2, a B cell-specific adaptor protein that has been reported to act as an alternative scaffold for PLC $\gamma 2(51,52)$.

We have shown that PLC $\gamma 2$ lipase activity is required for B cell spreading, and Btk-deficient DT40 B cells exhibit impaired spreading, presumably because of insufficient PLC $\gamma 2$ activation. However, as the absence of Btk does not result in the same magnitude of impairment in spreading as observed in the PLC $\gamma 2-\mathrm{KO}$ cells, we expect that PLC $\gamma 2$ activity may be regulated by alternative means. Indeed, PLC $\gamma 2$ can undergo Btk-independent phosphorylation at tyrosine 1217 (41), which may facilitate interaction with SH2 domaincontaining proteins residing within microsignalosomes and, thus, contribute to their stabilization. Surprisingly, we found no clear involvement of PI3K in the propagation of the spreading response in DT40, despite previous evidence reporting positive feedback mechanisms in which PLC $\gamma 2$ activation and phosphatidylinositol bisphosphate $\left(\mathrm{PIP}_{2}\right)$ hydrolysis is potentiated by PI3K activity and phosphatidylinositol $(3,4,5)$ triphosphate generation. $(53-56)$. It is possible that the spreading and contraction response only requires low levels of $\mathrm{PIP}_{2}$ hydrolysis, and amplification via PI3K may be more important later on to sustain signaling. We found that DT40 B cells lacking all three $\mathrm{IP}_{3} \mathrm{Rs}$ or PKC $\beta$ did not exhibit any major abnormalities in their spreading responses. The former observation is in contrast with the calcium dependence of receptor-induced spreading in $\mathrm{T}$ cells (57). This suggests that $\mathrm{B}$ cell spreading is either independent of $\mathrm{IP}_{3} \mathrm{R}$ and PKC $\beta$ activity, or that the presence of one is able to compensate for the absence of the other. There are several other diacylglycerol binding proteins in addition to $\mathrm{PKC} \beta$ that potentially act downstream of $\mathrm{PIP}_{2}$ hydrolysis. Of these, Ras guanine nucleotide releasing proteins have been implicated in BCR-induced Ras and extracellular signal-regulated kinase signaling in a PLC $\gamma 2$-dependent manner $(58,59)$. It seems likely that disruption of the PLC $\gamma 2$ activity can affect a whole range of downstream targets involved in cytoskeleton remodelling and/or signal amplification. PLC $\gamma 2$ may also directly modulate actin dynamics through the regulation of the activity or localization of actin-binding proteins such as cofilin $(60,61)$.

We have demonstrated that Vav is critical for the propagation of the B cell spreading response, and that this function of Vav is dependent on its GEF activity. The Vav family of proteins have been well established as regulators of cytoskeleton reorganization through their action as GEFs for small GTPases, such as Rho, Cdc42, and Rac, and other actin modulators, such as Wiskott-Aldrich syndrome protein (62). Therefore, it seems likely that one of the functions of Vav may involve the direct regulation of the actin cytoskeleton to ensure maximal contact area and generation of microsignalosomes. It is clear from SEM that Vav1/2-KO B cells are significantly impaired in their ability to spread on a bilayer and lack the lamellipodial extensions observed in WT B cells. As these cells are severely deficient in their ability to polymerize actin (63), it seems likely that the underlying cortical actin architecture is also compromised in these cells. Interestingly, a recent study has also implicated Vav-1 in the pathway regulating cellular cortical tension through deactivation of the ezrin-radixin-moesin family protein ezrin (64). Such deactivation, which is induced upon antigen receptor signaling in both $\mathrm{T}$ cells $(64,65)$ and B cells (66), causes disassembly of ezrin-radixin-moesin family proteins and cortical actin, and the resulting morphological deformation promotes cell-cell conjugation (64). This pathway may also be disrupted in Lyn-KO and Syk-KO B cells, as they too exhibit an unusual morphology, suggesting that they have underlying cytoskeletal abnormalities. Furthermore, these molecules may play a role in the direct regulation of cytoskeletal rearrangements and signal transduction events by mediating localization and phosphorylation of the cortactin homologue HS1 (67-69).

We observed that PLC 22 is recruited to antigen microclusters, even in the absence of Vav3, in accordance with data obtained in Vav-deficient Jurkat T cells (70). However, as the spreading response in these cells is limited, the total number of microsignalosomes generated is lower. We have also found that the relative frequency of PLC $\gamma 2$-containing microclusters generated is reduced in Vav3-KO DT40 B cells, suggesting that a synergistic relationship exists between these two components of the microsignalosome. Indeed, similar reductions in the total number and relative frequency of Vav-containing microsignalosomes were observed in PLC $\gamma 2-\mathrm{KO}$ cells. Previous papers have highlighted a potential feedback mechanism between Vav and PLC $\gamma 2$ activation and the control of calcium release $(44,71,72)$. In several cell types, including B cells and $\mathrm{T}$ cells, PLC $\gamma 2$ or PLC $\gamma 1$ phosphorylation, respectively, were 
attenuated in Vav-KO cells in response to receptor stimulation $(44,71,72)$. In view of the results presented in this paper, one could explain reduced PLC $\gamma 2$ phosphorylation levels in Vav-KO cells on the basis of (a) a reduction in the absolute numbers of microsignalosomes generated, because of restricted spreading, and (b) the relative frequency of effective microsignalosomes because of mislocalization or instability of its constituents. In these situations, imaging offers the opportunity of carrying out biochemistry in situ within single cells and, potentially, allows the resolution of differences that are masked or less apparent through classical biochemical characterization of whole cell populations stimulated by BCR cross-linking. To the best of our knowledge, a two-way synergistic relationship between PLC $\gamma 2$ and Vav has not previously been reported, and we propose that the integrated action of these two molecules during the spreading response ensures their most efficient functioning during the course of B cell activation.

We have very recently identified a novel and essential role for the B cell coreceptor CD19, independent of CD21, in response to membrane-bound antigen stimulation (29). CD19deficient $\mathrm{B}$ cells were found to be severely impaired in their capacity to spread and flux calcium after BCR engagement with membrane-bound antigen. In addition, CD19 was found to be transiently recruited to BCR-antigen microclusters, and it was suggested that it likely functions to amplify BCR signaling from within individual microclusters. We postulate that the synergy between PLC $\gamma 2$ and Vav demonstrated in this paper represents the molecular mechanism of BCR/ CD19 cooperation in response to BCR stimulation by membrane-bound antigen. Thus, during the recognition of membrane-bound antigen and the process of BCR-induced cell spreading, CD19 functions through the recruitment and activation of additional molecules of Vav, which integrate with signal transduction pathways from the BCR. Indeed, cocross-linking of BCR and CD19 via antibodies or by antigen tagged with complement lead to enhanced Vav activity (73). Intriguingly, Vav can interact with phosphorylated CD19, resulting in feedback on calcium signaling via phosphatidylinositol-4-phosphate 5-kinase activation and $\mathrm{PIP}_{2}$ synthesis, thus providing a potential source of PLC $\gamma 2$ substrate (74), and PLC $\gamma 2$ was also found to coprecipitate with CD19 (75). The role of CD19 in this proposed mechanism is highly homologous to that described for linker of activated $\mathrm{T}$ cells in the context of $\mathrm{T}$ cell activation. After engagement of the TCR, linker of activated $\mathrm{T}$ cells acts as an adaptor for the recruitment of intracellular signaling molecules that allow the propagation of signaling (76). This sophisticated coordination among the repertoire of signaling molecules ensures responses, in a contact-dependent manner, to an enormous potential range of antigen densities and affinities, allowing appropriate B cell activation.

\section{MATERIALS AND METHODS}

Cell preparation and culture. $\mathrm{Lyn}^{-1-}(\mathrm{Lyn}-\mathrm{KO}), \mathrm{Syk}^{-1-}(\mathrm{Syk}-\mathrm{KO})$, Btk $k^{-/-}$(Btk-KO), Blnk $k^{-/-}$(Blnk-KO), PLC $\gamma 2^{-/-}(\mathrm{PLC} \gamma 2-\mathrm{KO})$, Vav $^{-/-}$

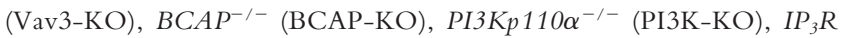

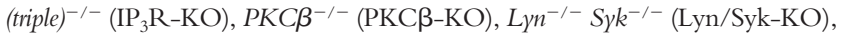
and WT DT40 B cells were used (31). All DT40 B cells, including stable transfectants, were maintained at $39.5^{\circ} \mathrm{C}$ in RPMI 1640 containing $10 \%$ fetal calf serum, $1 \%$ chicken serum (Invitrogen), penicillin and streptomycin antibiotics (Invitrogen), and $50 \mu \mathrm{M} 2$-mercaptoethanol (Sigma-Aldrich). The mouse strains used were PLC $\gamma 2^{\text {flox/flox }} \mathrm{CD} 19 \mathrm{Cre}^{+/-}(\mathrm{PLC} \gamma 2-\mathrm{KO})$ and PLC $\gamma 2^{+/+} \mathrm{CD} 19 \mathrm{Cre}^{+/-}(77,78)$. Vav1 $1^{-/-} \operatorname{Vav}^{-/-}(\operatorname{Vav} 1 / 2-\mathrm{KO})$ mice $(46)$ were provided by $\mathrm{M}$. Turner (Babraham Institute, Cambridge, UK) and subsequently crossed with transgenic MD4 mice (expressing HEL-specific BCR) (45). Mouse splenic naive B cells were purified by negative selection, as described previously (79); this resulted in a population enriched with $95-98 \%$ B cells. All experiments were approved by the Cancer Research UK Animal Ethics Committee and the UK Home Office.

Generation of PLC $\gamma 2$-enhanced GFP (EGFP) knock-in mice. The 3.1-kbp rat PLC $\gamma 2$ cDNA fragment from the unique EcoRI site to the end of the coding region was fused with EGFP cDNA from EGFP-N1 (Clontech Laboratories, Inc.), followed by a polyA signal cassette from pcDNA3.1 (Invitrogen). The 7.8-kbp upstream genomic fragment from the EcoRI site in exon 8 of the mouse PLC $\gamma 2$ gene was subcloned and ligated in frame to the EcoRI site of the rat PLC $\gamma 2-E G F P-p A$ fragment. The 2-kbp XhoI-BanIII genomic fragment flanking exon 9 of the mouse PLC $\gamma 2$ gene and the fragment described in the previous sentence were cloned into the pKS-TKNEO-LoxP vector (provided by K. Rajewsky, Harvard Medical School, Boston, MA) to obtain the targeting construct. The plasmid was linearized and transfected to $129 \mathrm{SvJ}$-derived embryonic stem cells and selected by G418 and ganciclovir. Targeted clones were injected into C57BL/6 blastocysts, and the obtained male chimeric mice were repeatedly crossed with female C57BL/6 mice to generate F1 mice with germline transmission of the PLC $\gamma 2-E G F P$ knock-in allele. The obtained heterozygotes were crossed with CAG-Cre transgenic mice (80) to remove the Neo cassette.

Constructs and transfections. PLC $\gamma 2$, PLC $\gamma 2-\mathrm{SH} 2$ and PLC $\gamma 2-\mathrm{LD}$, and Vav3-mGEF were generated by PCR, as previously described $(33,44,48)$. These constructs were fused to the C-terminus of GFP fused using the pENTR vector (Invitrogen) and inserted into the pApuro expression vector (48) by LR recombination using the Gateway system (Invitrogen). GFP-Syk in pcDNA3 and Vav1-EGFP (Vav-GFP) in EGFP-C1 (Clontech Laboratories, Inc.) were gifts from M. Reth (Max-Planck-Institute, Freiburg, Germany) and M. Turner, respectively. For transient transfections, $5 \times 10^{6}$ cells were nucleofected with the Nucleofector II system using $2 \mu \mathrm{g}$ DNA, nucleofection kit T, and program B-023 (Amaxa). To generate stable transfectants, $10^{7}$ cells were electroporated with $10 \mu \mathrm{g}$ DNA using a Gene Pulser (Bio-Rad Laboratories), and single clones were selected with $0.5 \mu \mathrm{g} / \mathrm{ml}$ puromycin.

Planar lipid bilayers. Planar lipid bilayers containing various antigens at the densities indicated in the figures were prepared in FCS2 chambers (Bioptechs) by liposome spreading, as previously described (79). In brief, Alexa Fluor 633-streptavidin (Invitrogen) was incorporated into the lipid bilayers and used to load bilayers with specific antigen. The monobiotinylated antigens used were anti-mouse $\mathrm{\kappa}$ clone HB-58 (81), anti-chicken IgM clone M1 (82), and HEL (Sigma-Aldrich). Assays were performed in chamber buffer (PBS, 0.5\% FCS, $2 \mathrm{mM} \mathrm{Mg}^{2+}, 0.5 \mathrm{mM} \mathrm{Ca}^{2+}, 1 \mathrm{~g} /$ liter D-glucose) at $37^{\circ} \mathrm{C}$.

$\mathrm{Ca}^{2+}$ influx assays on lipid bilayers. Intracellular $\mathrm{Ca}^{2+}$ influx measurements were performed as previously described (29). In brief, B cells labeled with $1 \mu \mathrm{M}$ Fluo-4FF AM (Invitrogen) were loaded onto antigen-containing lipid bilayers. This was performed in Hanks buffered saline solution supplemented with $1 \%$ FCS.

$\mathrm{Ca}^{2+}$ influx assays by flow cytometry. Intracellular $\mathrm{Ca}^{2+}$ influx measurements by flow cytometry were performed after labeling with $1 \mu \mathrm{M}$ indo-1 AM (Invitrogen), as previously described (29). 
Microscopy. All confocal microscopy, differential interference contrast (DIC), and IRM images were acquired with a microscope (Axiovert LSM 510; Carl Zeiss, Inc.), as previously described (23). PKH26 (Sigma-Aldrich) labeling of DT40 cell membranes was performed according to the manufacturer's instructions. To collect SEM images, cells were fixed on lipid bilayers with $2.5 \%$ gluteraldehyde $/ 4 \%$ paraformaldehyde and processed as previously described (23). TIRFM images were acquired with a CCD camera (Cascade II; Photonics) coupled to an inverted microscope (IX-81; Olympus). Images were recorded using Cell $\mathrm{R}$ software (Olympus), and analyzed and processed with the Volocity software package (Improvision) and ImageJ software (National Institutes of Health).

Image processing and data analysis. The contact area of the B cell with the artificial membrane and the amount of aggregated antigen were quantified using Volocity, measured in at least 20 cells of each type in a minimum of two independent experiments. The relative molecular enrichment in microclusters was determined using the surface plot option of the Image software. The migration of cells was measured manually using Volocity. Microclusters of antigen and GFP-tagged proteins were defined as an enrichment at least 1.5 times the fluorescence intensity of the background. Statistical significance was assessed by the Mann-Whitney test using Prism software (version 4.00c; GraphPad Software).

Online supplemental material. Fig. S1 represents intracellular calcium flux in WT DT40 B cells interacting with antigen-loaded lipid bilayers. Fig. S2 examines intracellular calcium flux in splenic B cells from WT and PLCg2-GFP knock-in (PLCg2-GFP KI) mice stimulated in solution. Fig. S3 shows confocal microscopy images of the spreading and contraction response for Blnk-KO, Btk-KO, and PI3K-KO DT40 B cells in comparison to WT DT40 B cells. Videos 1, 3, 4, and 9 are sequences of confocal microscopy images showing spreading, contraction, and antigen accumulation in WT DT40 (Video 1), Syk-KO DT40 (Video 3), PLCg2-KO DT40 (Video 4), and Vav3-KO DT40 (Video 9) B cells. Video 2 shows the recruitment of GFP-Syk to antigen microclusters in WT DT40 B cells, imaged by TIRFM. Video 5 and 6 are two examples of TIRFM videos showing the recruitment of PLCg2-GFP to antigen microclusters during spreading and contraction in primary splenic B cells from the knock-in mouse. Video 7 visualizes the recruitment of PLCg2-GFP to antigen microclusters in PLCg2-KO-reconstituted DT40 B cells. Video 8 shows the dynamics of the impaired spreading in Blnk-KO DT40 B cells through the visualization of antigen aggregation by TIRFM. Online supplemental material is available at http://www.jem .org/cgi/content/full/jem.20072619/DC1.

We thank Dr. Hideki Sanjo for his help in generating PLC 2 2-EGFP knock-in mice, Dr. Anne Weston for technical assistance with SEM, and Dr. Sebastian Fleire for provision of the Vav1/2-KO SEM image. We would also like to thank the members of Lymphocyte Interaction Laboratory and Mike Pütz for critical reading of the manuscript, and Mari Kurosaki for technical assistance.

This work was funded by Cancer Research UK.

The authors declare that they have no competing financial interests.

Submitted: 11 December 2007

Accepted: 27 February 2008

\section{REFERENCES}

1. Dal Porto, J.M., S.B. Gauld, K.T. Merrell, D. Mills, A.E. PughBernard, and J. Cambier. 2004. B cell antigen receptor signaling 101. Mol. Immunol. 41:599-613.

2. Reth, M., and J. Wienands. 1997. Initiation and processing of signals from the B cell antigen receptor. Annu. Rev. Immunol. 15:453-479.

3. DeFranco, A.L. 1997. The complexity of signaling pathways activated by the BCR. Curr. Opin. Immunol. 9:296-308.

4. Fruman, D.A., A.B. Satterthwaite, and O.N. Witte. 2000. Xid-like phenotypes: a B cell signalosome takes shape. Immunity. 13:1-3.

5. DeFranco, A.L. 2001. Vav and the B cell signalosome. Nat. Immunol. 2:482-484.
6. Kurosaki, T. 2002. Regulation of B-cell signal transduction by adaptor proteins. Nat. Rev. Immunol. 2:354-363.

7. Scharenberg, A.M., L.A. Humphries, and D.J. Rawlings. 2007. Calcium signalling and cell-fate choice in B cells. Nat. Rev. Immunol. 7:778-789.

8. Togni, M., K. Swanson, S. Reimann, S. Kliche, A. Pearce, L. Simeoni, D. Reinhold, J. Wienands, B. Neel, B. Schraven, and A. Gerber. 2005. Regulation of in vitro and in vivo immune functions by the cytosolic adaptor protein SKAP-HOM. Mol. Cell. Biol. 25:8052-8063.

9. Niiro, H., and E. Clark. 2002. Regulation of B-cell fate by antigenreceptor signals. Nat. Rev. Immunol. 2:945-956.

10. Vigorito, E., G. Bardi, J. Glassford, E. Lam, E. Clayton, and M. Turner. 2004. Vav-dependent and vav-independent phosphatidylinositol 3-kinase activation in murine $\mathrm{B}$ cells determined by the nature of the stimulus. J. Immunol. 173:3209-3214.

11. Brezski, R.J., and J.G. Monroe. 2007. B cell antigen receptor-induced Rac1 activation and Rac1-dependent spreading are impaired in transitional immature B cells due to levels of membrane cholesterol. J. Immunol. 179:4464-4472.

12. Kurosaki, T. 2002. Regulation of B cell fates by BCR signaling components. Curr. Opin. Immunol. 14:341-347.

13. Szakal, A.K., M.H. Kosco, and J.G. Tew. 1988. A novel in vivo follicular dendritic cell-dependent iccosome-mediated mechanism for delivery of antigen to antigen-processing cells. J. Immunol. 140:341-353.

14. Wu, J., D. Qin, G. Burton, A. Szakal, and J. Tew. 1996. Follicular dendritic cell-derived antigen and accessory activity in initiation of memory IgG responses in vitro. J. Immunol. 157:3404-3411.

15. Wykes, M., A. Pombo, C. Jenkins, and G. MacPherson. 1998. Dendritic cells interact directly with naive B lymphocytes to transfer antigen and initiate class switching in a primary $\mathrm{T}$-dependent response. J. Immunol. 161:1313-1319.

16. Balázs, M., F. Martin, T. Zhou, and J. Kearney. 2002. Blood dendritic cells interact with splenic marginal zone B cells to initiate T-independent immune responses. Immunity. 17:341-352.

17. Carrasco, Y.R., and F.D. Batista. 2007. B cells acquire particulate antigen in a macrophage-rich area at the boundary between the follicle and the subcapsular sinus of the lymph node. Immunity. 27:160-171.

18. Phan, T.G., I. Grigorova, T. Okada, and J.G. Cyster. 2007. Subcapsular encounter and complement-dependent transport of immune complexes by lymph node B cells. Nat. Immunol. 8:992-1000.

19. Junt, T., E. Moseman, M. Iannacone, S. Massberg, P. Lang, M. Boes, K. Fink, S. Henrickson, D. Shayakhmetov, N. Di Paolo, et al. 2007. Subcapsular sinus macrophages in lymph nodes clear lymph-borne viruses and present them to antiviral B cells. Nature. 450:110-114.

20. Batista, F.D., D. Iber, and M.S. Neuberger. 2001. B cells acquire antigen from target cells after synapse formation. Nature. 411:489-494.

21. Monks, C.R., B.A. Freiberg, H. Kupfer, N. Sciaky, and A. Kupfer. 1998. Three-dimensional segregation of supramolecular activation clusters in T cells. Nature. 395:82-86.

22. Grakoui, A., S. Bromley, C. Sumen, M. Davis, A. Shaw, P. Allen, and M. Dustin. 1999. The immunological synapse: a molecular machine controlling T cell activation. Science. 285:221-227.

23. Fleire, S.J., J.P. Goldman, Y.R. Carrasco, M. Weber, D. Bray, and F.D. Batista. 2006. B cell ligand discrimination through a spreading and contraction response. Science. 312:738-741.

24. Treanor, B., and F. Batista. 2007. Mechanistic insight into lymphocyte activation through quantitative imaging and theoretical modelling. Curr. Opin. Immunol. 19:476-483.

25. Bunnell, S.C., D.I. Hong, J.R. Kardon, T. Yamazaki, C.J. McGlade, V.A. Barr, and L.E. Samelson. 2002. T cell receptor ligation induces the formation of dynamically regulated signaling assemblies. J. Cell Biol. 158:1263-1275.

26. Campi, G., R. Varma, and M. Dustin. 2005. Actin and agonist MHCpeptide complex-dependent $\mathrm{T}$ cell receptor microclusters as scaffolds for signaling. J. Exp. Med. 202:1031-1036.

27. Yokosuka, T., K. Sakata-Sogawa, W. Kobayashi, M. Hiroshima, A. Hashimoto-Tane, M. Tokunaga, M. Dustin, and T. Saito. 2005. Newly generated $\mathrm{T}$ cell receptor microclusters initiate and sustain $\mathrm{T}$ cell activation by recruitment of Zap70 and SLP-76. Nat. Immunol. 6:1253-1262. 
28. Varma, R., G. Campi, T. Yokosuka, T. Saito, and M. Dustin. 2006. $\mathrm{T}$ cell receptor-proximal signals are sustained in peripheral microclusters and terminated in the central supramolecular activation cluster. Immunity. 25:117-127.

29. Depoil, D., S. Fleire, B. Treanor, M. Weber, K. Marchbank, V. Tybulewicz, M. Reth, N. Harwood, and F. Batista. 2008. CD19 is essential for B cell activation by promoting $\mathrm{B}$ cell receptor-antigen microcluster formation in response to membrane bound ligand. Nat. Immunol. 9:63-72.

30. Kurosaki, T. 1999. Genetic analysis of B cell antigen receptor signaling. Anпu. Rev. Immunol. 17:555-592.

31. Shinohara, H., and T. Kurosaki. 2006. Genetic analysis of B cell signaling. Subcell. Biochem. 40:145-187.

32. Rhee, S.G. 2001. Regulation of phosphoinositide-specific phospholipase C. Annu. Rev. Biochem. 70:281-312.

33. Patterson, R.L., D.B. van Rossum, N. Nikolaidis, D.L. Gill, and S.H Snyder. 2005. Phospholipase C-gamma: diverse roles in receptor-mediated calcium signaling. Trends Biochem. Sci. 30:688-697.

34. Fu, C., C. Turck, T. Kurosaki, and A. Chan. 1998. BLNK: a central linker protein in B cell activation. Immunity. 9:93-103.

35. Chiu, C.W., M. Dalton, M. Ishiai, T. Kurosaki, and A.C. Chan. 2002. BLNK: molecular scaffolding through 'cis'-mediated organization of signaling proteins. EMBO J. 21:6461-6472.

36. Ishiai, M., M. Kurosaki, R. Pappu, K. Okawa, I. Ronko, C. Fu, M. Shibata, A. Iwamatsu, A. Chan, and T. Kurosaki. 1999. BLNK required for coupling Syk to PLC gamma 2 and Rac1-JNK in B cells. Immunity. 10:117-125

37. Hashimoto, S., A. Iwamatsu, M. Ishiai, K. Okawa, T. Yamadori, M Matsushita, Y. Baba, T. Kishimoto, T. Kurosaki, and S. Tsukada. 1999. Identification of the SH2 domain binding protein of Bruton's tyrosine kinase as BLNK-functional significance of Btk-SH2 domain in B-cell antigen receptor-coupled calcium signaling. Blood. 94:2357-2364.

38. Wienands, J., J. Schweikert, B. Wollscheid, H. Jumaa, P. Nielsen, and M. Reth. 1998. SLP-65: a new signaling component in B lymphocytes which requires expression of the antigen receptor for phosphorylation. J. Exp. Med. 188:791-795.

39. Goitsuka, R., Y. Fujimura, H. Mamada, A. Umeda, T. Morimura, K. Uetsuka, K. Doi, S. Tsuji, and D. Kitamura. 1998. BASH, a novel signaling molecule preferentially expressed in B cells of the bursa of Fabricius. J. Immunol. 161:5804-5808.

40. Humphries, L.A., C. Dangelmaier, K. Sommer, K. Kipp, R.M. Kato, N. Griffith, I. Bakman, C.W. Turk, J.L. Daniel, and D.J. Rawlings. 2004. Tec kinases mediate sustained calcium influx via site-specific tyrosine phosphorylation of the phospholipase Cgamma Src homology 2-Src homology 3 linker. J. Biol. Chem. 279:37651-37661.

41. Kim, Y.J., F. Sekiya, B. Poulin, Y.S. Bae, and S.G. Rhee. 2004. Mechanism of B-cell receptor-induced phosphorylation and activation of phospholipase C-gamma2. Mol. Cell. Biol. 24:9986-9999.

42. Watanabe, D., S. Hashimoto, M. Ishiai, M. Matsushita, Y. Baba, T. Kishimoto, T. Kurosaki, and S. Tsukada. 2001. Four tyrosine residues in phospholipase C-gamma 2, identified as Btk-dependent phosphorylation sites, are required for $\mathrm{B}$ cell antigen receptor-coupled calcium signaling. J. Biol. Chem. 276:38595-38601.

43. Sugawara, H., M. Kurosaki, M. Takata, and T. Kurosaki. 1997. Genetic evidence for involvement of type 1, type 2 and type 3 inositol 1,4,5trisphosphate receptors in signal transduction through the B-cell antigen receptor. EMBO J. 16:3078-3088.

44. Inabe, K., M. Ishiai, A. Scharenberg, N. Freshney, J. Downward, and T. Kurosaki. 2002. Vav3 modulates B cell receptor responses by regulating phosphoinositide 3-kinase activation. J. Exp. Med. 195:189-200.

45. Goodnow, C.C., J. Crosbie, S. Adelstein, T.B. Lavoie, S.J. Smith-Gill, R.A. Brink, H. Pritchard-Briscoe, J.S. Wotherspoon, R.H. Loblay, K. Raphael, et al. 1988. Altered immunoglobulin expression and functional silencing of self-reactive B lymphocytes in transgenic mice. Nature. 334:676-682.

46. Doody, G.M., S.E. Bell, E. Vigorito, E. Clayton, S. McAdam, R. Tooze, C. Fernandez, I.J. Lee, and M. Turner. 2001. Signal transduction through Vav-2 participates in humoral immune responses and B cell maturation. Nat. Immunol. 2:542-547.
47. Jaffe, A.B., and A. Hall. 2005. Rho GTPases: biochemistry and biology. Annu. Rev. Cell Dev. Biol. 21:247-269.

48. Takata, M., H. Sabe, A. Hata, T. Inazu, Y. Homma, T. Nukada, H. Yamamura, and T. Kurosaki. 1994. Tyrosine kinases Lyn and Syk regulate $\mathrm{B}$ cell receptor-coupled $\mathrm{Ca} 2+$ mobilization through distinct pathways. EMBO J. 13:1341-1349.

49. Lowell, C.A., and P. Soriano. 1996. Knockouts of Src-family kinases: stiff bones, wimpy T cells, and bad memories. Genes Dev. 10:1845-1857.

50. Weng, W.K., L. Jarvis, and T.W. LeBien. 1994. Signaling through CD19 activates Vav/mitogen-activated protein kinase pathway and induces formation of a CD19/Vav/phosphatidylinositol 3-kinase complex in human B cell precursors. J. Biol. Chem. 269:32514-32521.

51. Foucault, I., S. Le Bras, C. Charvet, C. Moon, A. Altman, and M. Deckert. 2005. The adaptor protein 3BP2 associates with VAV guanine nucleotide exchange factors to regulate NFAT activation by the B-cell antigen receptor. Blood. 105:1106-1113.

52. de la Fuente, M.A., L. Kumar, B. Lu, and R.S. Geha. 2006. 3BP2 deficiency impairs the response of $\mathrm{B}$ cells, but not $\mathrm{T}$ cells, to antigen receptor ligation. Mol. Cell. Biol. 26:5214-5225.

53. Fluckiger, A.C., Z. Li, R.M. Kato, M.I. Wahl, H.D. Ochs, R. Longnecker, J.P. Kinet, O.N. Witte, A.M. Scharenberg, and D.J. Rawlings. 1998. Btk/Tec kinases regulate sustained increases in intracellular $\mathrm{Ca} 2+$ following $\mathrm{B}$-cell receptor activation. EMBO J. 17:1973-1985.

54. Scharenberg, A.M., O. El-Hillal, D.A. Fruman, L.O. Beitz, Z. Li, S. Lin, I. Gout, L.C. Cantley, D.J. Rawlings, and J.P. Kinet. 1998. Phosphatidylinositol-3,4,5-trisphosphate (PtdIns-3,4,5-P3)/Tec kinasedependent calcium signaling pathway: a target for SHIP-mediated inhibitory signals. EMBO J. 17:1961-1972.

55. Falasca, M., S. Logan, V. Lehto, G. Baccante, M. Lemmon, and J. Schlessinger. 1998. Activation of phospholipase C gamma by PI 3-kinase-induced $\mathrm{PH}$ domain-mediated membrane targeting. EMBO J. 17:414-422.

56. Bolland, S., R. Pearse, T. Kurosaki, and J. Ravetch. 1998. SHIP modulates immune receptor responses by regulating membrane association of Btk. Immunity. 8:509-516.

57. Bunnell, S.C., V. Kapoor, R.P. Trible, W. Zhang, and L.E. Samelson. 2001. Dynamic actin polymerization drives T cell receptor-induced spreading: a role for the signal transduction adaptor LAT. Immunity. 14:315-329.

58. Oh-hora, M., S. Johmura, A. Hashimoto, M. Hikida, and T. Kurosaki. 2003. Requirement for Ras guanine nucleotide releasing protein 3 in coupling phospholipase C- $\gamma 2$ to Ras in B cell receptor signaling. J. Exp. Med. 198:1841-1851.

59. Coughlin, J.J., S.L. Stang, N.A. Dower, and J.C. Stone. 2005. RasGRP1 and RasGRP3 regulate B cell proliferation by facilitating B cell receptor-Ras signaling. J. Immunol. 175:7179-7184.

60. Yonezawa, N., Y. Homma, I. Yahara, H. Sakai, and E. Nishida. 1991 A short sequence responsible for both phosphoinositide binding and actin binding activities of cofilin. J. Biol. Chem. 266:17218-17221.

61. DesMarais, V., M. Ghosh, R. Eddy, and J. Condeelis. 2005. Cofilin takes the lead. J. Cell Sci. 118:19-26.

62. Hornstein, I., A. Alcover, and S. Katzav. 2004. Vav proteins, masters of the world of cytoskeleton organization. Cell. Signal. 16:1-11.

63. Holsinger, L.J., I.A. Graef, W. Swat, T. Chi, D.M. Bautista, L. Davidson, R.S. Lewis, F.W. Alt, and G.R. Crabtree. 1998. Defects in actin-cap formation in Vav-deficient mice implicate an actin requirement for lymphocyte signal transduction. Curr. Biol. 8:563-572.

64. Faure, S., L. Salazar-Fontana, M. Semichon, V. Tybulewicz, G. Bismuth, A. Trautmann, R. Germain, and J. Delon. 2004. ERM proteins regulate cytoskeleton relaxation promoting $\mathrm{T}$ cell-APC conjugation. Nat. Immunol. 5:272-279.

65. Delon, J., K. Kaibuchi, and R. Germain. 2001. Exclusion of CD43 from the immunological synapse is mediated by phosphorylation-regulated relocation of the cytoskeletal adaptor moesin. Immunity. 15:691-701.

66. Gupta, N., B. Wollscheid, J. Watts, B. Scheer, R. Aebersold, and A. DeFranco. 2006. Quantitative proteomic analysis of B cell lipid rafts reveals that ezrin regulates antigen receptor-mediated lipid raft dynamics. Nat. Immunol. 7:625-633. 
67. Yamanashi, Y., T. Fukuda, H. Nishizumi, T. Inazu, K. Higashi, D. Kitamura, T. Ishida, H. Yamamura, T. Watanabe, and T. Yamamoto. 1997. Role of tyrosine phosphorylation of HS1 in B cell antigen receptor-mediated apoptosis. J. Exp. Med. 185:1387-1392.

68. Hao, J.J., G.B. Carey, and X. Zhan. 2004. Syk-mediated tyrosine phosphorylation is required for the association of hematopoietic lineage cell-specific protein 1 with lipid rafts and B cell antigen receptor signalosome complex. J. Biol. Chem. 279:33413-33420.

69. Gomez, T.S., S.D. McCarney, E. Carrizosa, C.M. Labno, E.O. Comiskey, J.C. Nolz, P. Zhu, B.D. Freedman, M.R. Clark, D.J Rawlings, et al. 2006. HS1 functions as an essential actin-regulatory adaptor protein at the immune synapse. Immunity. 24:741-752.

70. Braiman, A., M. Barda-Saad, C. Sommers, and L. Samelson. 2006 Recruitment and activation of PLCgamma1 in T cells: a new insight into old domains. EMBO J. 25:774-784.

71. Manetz, T.S., C. Gonzalez-Espinosa, R. Arudchandran, S. Xirasagar, V. Tybulewicz, and J. Rivera. 2001. Vav1 regulates phospholipase cgamma activation and calcium responses in mast cells. Mol. Cell. Biol. 21:3763-3774.

72. Reynolds, L.F., L.A. Smyth, T. Norton, N. Freshney, J. Downward, D. Kioussis, and V.L. Tybulewicz. 2002. Vav1 transduces T cell receptor signals to the activation of phospholipase $\mathrm{C}-\gamma 1$ via phosphoinositide 3-kinase-dependent and -independent pathways. J. Exp. Med. 195:1103-1114.

73. Fearon, D.T., and M.C. Carroll. 2000. Regulation of B lymphocyte responses to foreign and self-antigens by the CD19/CD21 complex. Annu. Rev. Immunol. 18:393-422.
74. O'Rourke, L.M., R. Tooze, M. Turner, D.M. Sandoval, R.H. Carter, V.L. Tybulewicz, and D.T. Fearon. 1998. CD19 as a membraneanchored adaptor protein of B lymphocytes: costimulation of lipid and protein kinases by recruitment of Vav. Immunity. 8:635-645.

75. Brooks, S.R., P.M. Kirkham, L. Freeberg, and R.H. Carter. 2004. Binding of cytoplasmic proteins to the CD19 intracellular domain is high affinity, competitive, and multimeric. J. Immunol. 172:7556-7564.

76. Zhang, W., and L. Samelson. 2000. The role of membrane-associated adaptors in T cell receptor signalling. Semin. Immunol. 12:35-41.

77. Hashimoto, A., K. Takeda, M. Inaba, M. Sekimata, T. Kaisho, S Ikehara, Y. Homma, S. Akira, and T. Kurosaki. 2000. Cutting edge essential role of phospholipase C-gamma 2 in B cell development and function. J. Immunol. 165:1738-1742.

78. Rickert, R.C., J. Roes, and K. Rajewsky. 1997. B lymphocyte-specific, Cre-mediated mutagenesis in mice. Nucleic Acids Res. 25:1317-1318.

79. Carrasco, Y.R., S.J. Fleire, T. Cameron, M.L. Dustin, and F.D. Batista. 2004. LFA-1/ICAM-1 interaction lowers the threshold of B cell activation by facilitating $\mathrm{B}$ cell adhesion and synapse formation. Immunity. 20:589-599.

80. Sakai, K., and J. Miyazaki. 1997. A transgenic mouse line that retains Cre recombinase activity in mature oocytes irrespective of the cre transgene transmission. Biochem. Biophys. Res. Commun. 237:318-324.

81. Brumeanu, T.D., S. Casares, A. Bot, S. Bot, and C.A. Bona. 1997. Immunogenicity of a contiguous $\mathrm{T}-\mathrm{B}$ synthetic epitope of the A/PR/ 8/34 influenza virus. J. Virol. 71:5473-5480.

82. Chen, C.L., J.E. Lehmeyer, and M.D. Cooper. 1982. Evidence for an IgD homologue on chicken lymphocytes. J. Immunol. 129:2580-2585. 